\title{
La métamorphose de Yurupari : flûtes, trompes et reproduction rituelle dans le Nord-Ouest amazonien
}

\section{Dimitri Karadimas}

\section{(2) OpenEdition}

\section{Journals}

Édition électronique

URL : https://journals.openedition.org/jsa/9253

DOI : 10.4000/jsa.9253

ISSN : 1957-7842

Éditeur

Société des américanistes

Édition imprimée

Date de publication : 15 juillet 2008

Pagination : 127-169

ISSN : 0037-9174

Référence électronique

Dimitri Karadimas, "La métamorphose de Yurupari : flûtes, trompes et reproduction rituelle dans le Nord-Ouest amazonien », Journal de la Société des américanistes [En ligne], 94-1 | 2008, mis en ligne le 10 juin 2013, consulté le 02 septembre 2022. URL : http://journals.openedition.org/jsa/9253 ; DOI : https://doi.org/10.4000/jsa.9253 


\title{
LA MÉTAMORPHOSE DE YURUPARI : FLÛTES, TROMPES ET REPRODUCTION RITUELLE DANS LE NORD-OUEST AMAZONIEN
}

\author{
Dimitri KARADIMAS *
}

\begin{abstract}
Dans cet article, nous proposons d'analyser les différentes figures mythiques présentes dans l'Amazonie du Nord-Ouest sous le nom de Yurupari. En comparant des données issues de l'ethnographie miraña avec celles des groupes tukano du Vaupès et des groupes Arawak du nord de cette zone, il apparaît que ces figures renvoient, toutes, à une guêpe solitaire qui se reproduit en se servant de ses proies à la fois comme réceptacle et nourriture pour sa descendance puisque la proie est mangée de l'intérieur par la larve de la guêpe. Ce comportement parasitoïde est anthropomorphisé par ces populations sous la forme d'une insémination. Avec certaines caractéristiques et composantes de l'hyménoptère, ce comportement sert de référent au rituel d'initiation masculin et à l'interdit pour les femmes de voir les flûtes sacrées, construisant ainsi idéologiquement le corps de chacun des sexes. Cette contribution explore l'ensemble des implications mythologiques et idéologiques de cette identification. [Mots-clés : métamorphose, Amazonie du Nord-Ouest, sexualité, analogie visuelle, rite d'initiation, flûtes sacrées.]
\end{abstract}

La metamorfosis de Yurupari : flautas, trompetas y reproducción ritual en el Noroeste Amazónico. En este artículo, el autor propone un análisis de las diferentes figuras míticas presentes en la Amazonía del Noroeste bajo el nombre de Yurupari. Comparando unos datos de la etnografía miraña con los de grupos tukano del Vaupés y de los grupos arawak del norte de esta zona, aparece que una avispa solitaria es el elemento común de estas figuras míticas. Ésta se reproduce usando sus presas al mismo tiempo como receptáculo y como comida para su descendencia. Cuando las poblaciones del Noroeste antropomorfisan este comportamiento seudo-parasítico, lo describen como una inseminación. Con otras características y componentes del himenóptero, este comportamiento sirve de referente tanto al ritual de iniciación masculino como a la interdicción de ver las flautas sagradas que existe para las mujeres, construyendo así ideológicamente el cuerpo de cada uno de los sexos. La contribución expone el conjunto de las implicaciones mitológicas e ideológicas de esta identificación. [Palabras claves :

* CNRS/Laboratoire d'anthroplogie sociale, 52 rue du Cardinal Lemoine, 75005 Paris [dimitri.karadimas@college-de-france.fr].

Journal de la Société des Américanistes, 2008, 94-1, pp. 127-169. O Société des Américanistes. 
metamorfosis, indígenas del noroeste amazónico, sexualidad, analogía visual, rito de iniciación, flautas sagradas.]

The metamorphosis of Yurupari: flutes, trumpets and ritual reproduction in the Northwest Amazon. In this article, the author proposes to analyse different mythological figures known in the North-West Amazon as Yurupari. Making a comparison between some ethnographical data from the Miraña, from the Tukano speaking groups of the Vaupes and from the Arawak groups north of the area, it appears that a solitary wasp is the common element of these mythological figures. This wasp reproduces itself using their preys as a receptacle and as food for its offspring. When anthropomorphized by the North-West Amazon populations, this pseudo-parasitical behaviour is seen as an insemination. Along with other characteristics and components from the hymenopter, this behaviour is an essential referent as much for the male initiation ritual as for the prohibition to see the sacred flutes that exists for women, thus constructing ideologically the bodies of both sexes. The contribution will explore the major mythological and ideological implications of this identification. [Key words : metamorphosis, NorthWest Amazon, sexuality, visual analogy, initiation ritual, sacred flutes.]

Les études sur le complexe rituel de Yurupari sont légion dans l'ethnologie du Nord-Ouest amazonien. Depuis la publication en 1890 de Stradelli sur les premiers éléments du mythe jusqu'à la toute récente présentation des données cubeo par Goldman (2004), en passant par les différentes analyses - et débats - entre Stephen Hugh-Jones (1979) et Gerardo Reichel-Dolmatoff (1996), il pourrait sembler difficile d'ajouter un élément novateur au dossier. Cet essai se propose pourtant d'être la première tentative à visée comparative de rassembler les différentes modalités idéologiques liées au rituel de Yurupari dans le Nord-Ouest amazonien. Je me pencherai tout particulièrement sur le processus naturel de la métamorphose, notamment celui des insectes comme modèles de la reproduction sociale humaine.

Pour la résumer rapidement, on peut dire que la problématique générale de la mythologie de Yurupari renvoie à celle de la domination des hommes sur les femmes, après les avoir dépossédées des trompes et/ou des flûtes « sacrées ». En effet, dans les mythes, les femmes étaient, à l'origine, les premières à posséder les flûtes et pouvaient, comme le font aujourd'hui les hommes, engendrer une descendance grâce à elles. De plus, elles les utilisaient comme objets de plaisir. Les hommes étaient alors soumis à la volonté des femmes et devaient accomplir leurs tâches (Hugh-Jones 1979, p. 266). L'acte civilisateur masculin fut de subtiliser les flûtes aux femmes et de leur imposer la gestation. Et, grâce aux flûtes, l'engendrement extra-utérin ne fut plus réservé qu'aux hommes.

Toutefois, la tradition de Yurupari est plus que cela : elle est non seulement intimement connectée à l'idée de distinction de genre, mais aussi à la notion même de sexualité. 
Pour les populations du Nord-Ouest amazonien, le rituel et la mythologie qui l'accompagne sont d'une importance primordiale tant dans la construction des genres que dans la création d'interdits. Dans le même esprit, les interdits permettent d'instaurer une domination garantissant la séparation des sexes - plus dans l'idéologie que dans les faits d'ailleurs, une sorte de modus vivendi ${ }^{1}$. Pour ce faire, le rituel et la mythologie de Yurupari utilisent une profusion d'images - aussi bien dans le registre du langage que dans celui de l'action rituelle - puisées le plus souvent dans l'environnement. Ces référents environnementaux accréditent, pour les membres de ces sociétés, une construction de l'image des corps masculin et féminin et un mode de sexualité.

Tout ce qui vient d'être décrit est présent particulièrement dans les groupes Tukano du Vaupès, mais pas seulement. Ainsi les Miraña, groupe avec lequel j'ai travaillé ${ }^{2}$ et grâce auquel j'ai recueilli les données ethnographiques constituant la première partie de ce travail, ne font pas partie de l'ensemble culturel précédent et ne peuvent pas être rattachés au groupe linguistique des Tukano orientaux ou occidentaux. Il en va de même pour les Uitoto, voisins des Miraña, mais qui n'entretiennent que peu de rapports, voire aucun, avec les groupes du Vaupès central. De fait, ce complexe rituel et mythologique s'étend très largement au-delà de l'aire du Vaupès, puisque l'on retrouve l'utilisation des flûtes et des trompes interdites à la vue des non-initiés - et plus particulièrement des femmes -, jusque chez les Tikuna de l'Amazone au sud de la Colombie (Goulard 1998 et communication personnelle), ainsi que chez les Wakuénai du Guainía au nord du Vaupès (Hill 2001), pour ne citer que les cas les plus éloignés de l'épicentre de cette aire.

Les conclusions et les déductions présentées ici ne sont à retenir que pour les Miraña, du moins en ce qui concerne la première partie. Toutefois, en tentant une comparaison des données miraña avec celles issues des ethnographies d'autres sociétés de la région, il est possible de dégager un tableau général cohérent. Les données miraña et uitoto n'entrent pas en contradiction avec celles de la zone tukano, mais semblent plutôt les compléter. Aussi, la comparaison avec les éléments ethnographiques provenant des groupes de langues tukano me semble essentielle. J'aurai donc recours essentiellement à l'ouvrage de Reichel-Dolmatoff (1996) - notamment pour les quatre énoncés mythiques liés au complexe de Yurupari - et au travail réalisé par Stephen Hugh-Jones (1979) sur les Barasana. Il n'en reste pas moins que la compréhension plus générale de ce complexe rituel du Nord-Ouest amazonien devrait, pour rendre compte de toutes les spécificités culturelles, inclure l'ensemble des conclusions émises par ces auteurs ${ }^{3}$. Seul l'aspect de la mythologie est ici abordé, réservant l'analyse des phases du rituel d'initiation pour une autre étude.

Si nous adoptons à présent un point de vue comparatif à propos du type d'organisation sociale dans cette aire culturelle, il apparaît que cette région représente à bien des égards une exception pour l'Amazonie indigène : en la comparant par exemple avec le Brésil central ou encore à la zone guyanaise, la 
notion d'ancestralité semble liée à la présence de groupes d'unifiliation patrilinéaires. Dans ces sociétés, le rite de Yurupari sert surtout à l'initiation masculine. Il permet de construire des hommes issus, si possible, de personnes masculines, c'est-à-dire en excluant toute contribution féminine, donc des affins. Le complexe de Yurupari a donc lieu dans des sociétés où la notion de " groupes d'unifiliation " est beaucoup plus présente par rapport à l'aspect plus cognatique des autres aires amazoniennes (Hugh-Jones 2001, p. 246).

Tout en jouant sur l'ostentation rituelle d'une manipulation des corps des initiés masculins, cette construction de la masculinité se fait par retranchement de pratiques sexuelles liées au corps des femmes.

La méthodologie utilisée s'appuie sur l'analyse des textes, des métaphores et autres tropes liés aux termes employés. Cette méthodologie repose aussi sur une théorie plus générale de l'image et de son évocation : un artefact peut être employé dans un rituel ou nommé dans un mythe non pas pour ce qu'il est, ni pour sa matière, ni pour son essence, mais bien pour l'image qu'il est censé générer dans l'esprit des participants à un rituel ou des auditeurs d'une narration. Je rejoins ici un des points évoqués par Reichel-Dolmatoff (1996, pp. xxxi, xxxii) à propos des textes desana : "If the Tukanoans strech analogies in a way uncommon to our logic, the task must consist in identifying the "likeness" of things, expressed in the word dohpállike, similar to », et par Hill (1993) dans son analyse métaphorique des chants liés à la mythologie de Kuwai, l'équivalent wakuenai de Yurupari.

Revenons rapidement sur l'utilisation du code sexuel dans les groupes du Nord-Ouest amazonien. Reichel-Dolmatoff (ibid.) l'utilise de façon abondante, parfois peut-être de façon excessive. D'autres travaux ethnographiques (Hugh-Jones ibid. ; Karadimas 2005) ont montré que ce « code » devenait particulièrement intéressant si on le considérait sous son aspect corporel et pas seulement sexuel. J'aborderai donc l'ensemble des images du corps auquel les groupes du Nord-Ouest ont recours pour construire leur système de connaissance (Karadimas 2005).

Je présenterai, dans un premier temps, les données miraña sur le complexe du Yurupari pour montrer comment est pensé le processus d'engendrement et, donc, la construction des sexes. Puis, en comparant ces données avec celles présentées dans d'autres travaux, nous verrons comment elles se rendent mutuellement intelligibles.

\section{LES DONNÉES MIRAÑA}

À propos des interdits liés aux flûtes et aux trompes ${ }^{4}$, un de mes informateurs miraña me faisait remarquer que s'il n'y avait pas « Yurupari », il n'y aurait tout simplement pas d'enfant, tout comme il n'y aurait pas de rapports sexuels. Il faut 
donc se pencher sur le processus de fécondation tel qu'il est compris par les Miraña.

Dans la mythologie miraña, le processus de fécondation renvoie à la première humanité sur terre (dont l'humanité actuelle n'est pas issue). L'humanité primordiale est issue d'un lombric " pensé » par le créateur, qui rentrait et sortait de la terre pour rester dans cette première matrice terrestre. Ce Lombric divin, appelé le « Lombric de notre Création » (mè piíßénè tó'hù), représente successivement le sexe du créateur et l'enfant à naître et est attiré par le sang menstruel des femmes. C'est lui qui va former l'enfant dans la matrice. Si ce lombric n'existait pas, disent les Miraña, un enfant ne pourrait pas se former. Il représente aussi une âme qui prend possession de la matrice. En ce sens, Lombric n'est actuellement qu'une représentation d'une âme qui va se matérialiser dans un enfant. Il ne s'agirait pas d'un véritable lombric qui entre dans la matrice ; c'est le membre masculin qui lui est comparable.

Le sperme est aussi considéré comme de la " bave ou salive de Yurupari » (ñóñái tógwà, "sueur de Yurupari/lombric »), c'est-à-dire de la " viscosité de lombric » (tółhù rérékò) qui est interprétée par les Miraña comme du sperme divin. Toujours selon le discours mythique, le "lombric», attiré par le sang menstruel, " entre » dans la matrice et prépare, avec cette salive, l'endroit où l'enfant va se loger. Tant que ce lombric n'a pas préparé la matrice, une femme ne peut pas être fécondée.

Selon les Miraña, le fait que les lombrics soient attirés par le sang menstruel oblige les femmes qui ont leurs règles à accrocher leur hamac en hauteur pour éviter qu'un véritable lombric ne sorte de terre et ne vienne sucer leur sang pendant la nuit. Il est également déconseillé aux femmes qui allaitent de dormir la face contre terre au risque de voir les lombrics venir téter leurs seins.

C'est donc autour de cette figure de Lombric que se construit, chez les Miraña, le personnage désigné communément dans le Nord-Ouest comme Yurupari.

Dans l'utérus se trouvent l'enfant (tstimé), le liquide amniotique, le placenta (tsíménè mí'ò, " peau rigide de l'enfant » ou tsíménè dò'póhì, un type de galette de cassave) qui est aussi l'esprit, « l'ombre » qu'a formé le lombric dans la matrice et, enfin, le cordon ombilical (íhúbá, « boyau, viscère de la bouche du bas ») à partir duquel l'enfant se nourrit du sang de sa mère. Selon un informateur, ce serait le lombric qui formerait ce cordon et qui continuerait de « sucer » le sang de la mère pour l'enfant.

Le « lombric » est tout à la fois le pénis du père et « l'ombre » de l'enfant qui est constituée du placenta et du cordon ombilical ; on peut donc constater une surenchère d'éléments symboliques sur ce seul lombric. Qui représente quoi dans cette profusion d'éléments?

Il semble possible de faire une certaine part des choses. Si on considère que le lombric entre dans la matrice pour la « préparer » afin d'accueillir l'enfant et que 
sa « bave » est perçue comme du sperme, il est licite de retranscrire cette formulation mythique comme le pénis et le sperme du père. Parallèlement, ce lombric reste dans la matrice pour "former » l'enfant. Certains des interdits sexuels concernant le chasseur qui vient de manger une pièce de gibier lui prescrivent de ne pas coucher avec sa femme ou de ne pas toucher ses enfants avec sa main : son sperme et sa sueur sont alors « chargés » de « l'odeur » de ce gibier (et ses enfants, présents ou à venir, pourraient devenir " animal »). Or il semble que le seul élément qui reste dans la matrice au moment de l'acte sexuel soit du sperme ; mais ce sperme contient l'âme et l'ombre (nààßénè) de ce gibier. Il semble donc que le « lombric » et sa salive ou bave soient en fait le spectre, le nàà̉énè de l'animal ingéré par le père qui cherche de la sorte à se réincarner. C'est lui qui va former le " tsíménè nààßénè » c'est-à-dire l'esprit, "l'ombre » de l'enfant et qui va le nourrir à partir du sang de la mère. Le placenta est donc « l'ombre de l'enfant » mais, parallèlement, cette « ombre » ou ce « spectre » provient d'une qualité du sperme du père qui a mangé du gibier.

Pour interpréter un épisode d'un mythe uitoto (Preuss 1921, p. 75), on peut suivre la même direction. À la suite d'une piqûre d'épine, l'âme d'un personnage décapité pénètre sous forme de larve dans l'articulation du genou de son meurtrier, non pas pour renaître ou pour former une descendance à son image comme il l'aurait fait dans une matrice, mais pour faire mourir son meurtrier en corrompant sa jambe. En ce sens, l'âme est une larve décrite comme un « lombric » et ce qui entre dans la matrice subit une métamorphose comparable à une mue d'insecte, la salive de cette larve filant un fil de soie pour former un cocon (la matrice).

Il est ainsi possible de poursuivre les associations symboliques concernant le lombric. Il est celui qui suce le sang menstruel de la mère pour nourrir l'enfant par le nombril (la « bouche du bas »); il est aussi celui qui tète la mère pour continuer à nourrir l'enfant, une fois celui-ci sorti du ventre de sa mère. Le terme miraña pour désigner aussi bien le pénis que le lombric est ñóñá'i. Si lombric est un "téteur", il tète (ñónóói) la mère par l'intérieur, et par l'extérieur une fois l'enfant mis au monde : le terme qui désigne Yurupari, lombric (ñóñái i) semble être construit sur ñóñó’ « téter». De là, les conseils donnés aux femmes qui allaitent leurs enfants de ne pas faire pendre leurs seins vers la terre au risque qu'un véritable lombric vienne les téter. En ce sens, il est possible d'affirmer que Lombric, après avoir passé une partie de son existence qui se termine avec le placenta, poursuit celle-ci avec l'enfant, ce qui expliquerait qu'il soit aussi désigné, dans les discours des Miraña, comme un enfant et, ce, conjointement au pénis du créateur. Lombric est tout à la fois - mais successivement - le pénis, l'ensemble placenta-cordon ombilical et l'enfant qui naît. Autrement dit, Lombric est un être changeant qui se transforme et subit des mues. Il semble ainsi que Lombric représente pour l'humanité ce qu'une larve réalise pour les insectes. Ce dernier point découle du fait que, dans le mythe uitoto signalé plus haut, l'âme du 
père de Souffleur réapparaît comme larve et cherche à pénétrer un équivalent de matrice (Karadimas 2003). Le lombric et la larve apparaissent donc dans une position similaire. Le cocon dans lequel la larve se transforme en chrysalide, puis en insecte, serait la matrice ; ce qui restera de la chrysalide, l'exuvie, est comparable au placenta humain. La succession lombric (chenille-larve)/cocon/ chrysalide/imago est ainsi équivalente à celle de pénis (sperme-âme)/matrice/ placenta/foetus propre aux humains. Pour le dire autrement, « l'ombre »-dans le sens de spectre -, le nààßénè d'un insecte renvoie à sa mue, à son exuvie (míò) ; pour le nourrisson, c'est le placenta avec la membrane amniotique qui est sa mí 0 .

Il est possible de retrouver ces éléments dans le mythe de la création miraña. En effet, les éléments métaphoriques évoqués pour rendre compte de la première terre renvoient aussi bien au modèle de l'escargot qu'à celui de la chrysalide : ils sont nécessaires pour comprendre à quoi sont comparées les trompes du rituel.

\section{LA PROTO-CRÉATION MIRAÑA}

Dans le mythe miraña évoquant la création de la terre première, différente de l'actuelle, il est fait référence à un processus de métamorphose.

Issu du néant, l'ensemble des premières phases de la création est présenté comme un processus physiologique relatif à la procréation. L'acte initial décrit est de « ficher » ce qui sera la première terre, c'est-à-dire de la « planter » ou, bien plus, qu'elle se fiche elle-même. Le verbe u'pétsò exprime l'action de «ficher », comme celle de planter une bouture de manioc dans la terre. Cette action peut aussi renvoyer à celle d'une chrysalide qui « fiche » son extrémité inférieure dans un coussin de soie qu'elle a tissé sur une branche. Son extrémité inférieure est garnie de petits dards qui se plantent et lui permettent de se maintenir suspendue : dans le mythe, la proto-création est comparée à cette action.

Dans la suite du mythe, il est question d'un « enveloppement » du monde : l'utilisation du lexème tó àrádè souligne le caractère sexuel, agricole et végétal d'une semence ou d'une épine plantée et contenue. La transformation s'effectue ensuite rapidement : la " terre » du créateur prend une position accroupie ou fotale, puis la forme d'un escargot dont la coquille en colimaçon est comparée par les Miraña à l'enroulement de l'écorce d'une trompe de Yurupari (útíhádji). Ces éléments font référence à l'image d'une chrysalide. L'abdomen par l'extrémité duquel elle est suspendue rappelle une coquille d'escargot car il est divisé en plusieurs segments de taille croissante et s'élargit de façon conique. Le son que produit cette " conque » mythique, terre du créateur, est similaire à celui des trompes. Cet escargot est ainsi comme le Yurupari du monde sur lequel vient prendre place le créateur. Le son de la trompe semble augmenter en même temps que la coquille qui va former le lieu sur lequel le créateur prendra place. Le créateur prend corps dans la coquille de cet escargot - formulation imagée qui ne 
manque pas d'évoquer ce qui se réalise à l'intérieur de la chrysalide -, puis sort de celle-ci et prend place sur elle. L'exuvie devient sa première " terre ". Chez les Miraña, dans le rituel de Yurupari, la trompe fait penser à une chrysalide. Dans ce mythe, la première manifestation du démiurge se fait par la voix qui duplique celle du son produit par une « coquille d'escargot » et qui peut être comparé à celui des trompes de Yurupari. Le son des trompes est comme un son de conque : il donne naissance. Le créateur n'existe que par ce son qui est sa voix. La suite du mythe décrit le développement de ce fœtus divin dans ce qui apparaît comme une matrice cosmique ou, pour le moins, une " proto-matrice », c'est-à-dire un lieu de gestation sans corps, un « sac de gestation » sans corps pour le contenir.

Proposer une interprétation de cette proto-création est une tâche difficile. Le démiurge apparaît d'abord comme un fœtus en gestation ; dans la suite du mythe, le premier dialogue du démiurge a lieu avec Lombric qui se trouve sur une terre indépendante de celle où réside le créateur. Lombric (il faudrait en fait traduire par Larve) peut ainsi prendre la valeur d'un sexe masculin ou celle d'un cordon ombilical ou encore celle d'un enfant. Le fait de représenter Lombric sous les traits de Yurupari évoque cette multiplicité de sens puisque les trompes de Yurupari correspondent, pour les Miraña, à « la transformation d'un enfant "brillant" qui, lorsqu'il est frappé contre terre, prend l'apparence des trompes » ou sort des trompes. Lombric, sous les traits de Yurupari, est considéré par les Miraña comme le sexe du créateur ou une émanation de celui-ci qui prend la forme d'une âme venant se loger dans un « ventre » sous la forme d'une larve. Cette multiplicité des identités apparaît également chez les Barasana où le rituel de Yurupari tient une place importante (Hugh-Jones 1979). Christine HughJones donne l'interprétation suivante pour les Barasana qui relient la première terre à un placenta primordial :

Le placenta enterré illustre plusieurs propriétés de l'univers-comme-matrice: un énorme ver blanc en sort, qui perce les couches de l'univers jusqu'à ce que SoleilPrimordial lui brûle la tête. En raison de cette brûlure, il est appelé Le-Décapité (Rihoa $M a n g u)$, un personnage dépeint dans d'autres contextes comme un homme sans tête, le mari de Romi Kumu. La relation sexuelle entre Romi Kumu et Le-Décapité est ainsi transposée sur une échelle cosmique: l'hémisphère inférieur est semblable à une matrice contenant un placenta décomposé, et l'axe vertical, le pénis ou l'enfant, est fait par le passage vertical du ver. (ibid., p. 267; italique et traduction de l'auteur, D. K.)

Ainsi, en gardant la vision placentaire de la première terre, c'est-à-dire l'escargot - image de l'exuvie - pour le mythe miraña, la relation du démiurge à cette « terre » serait équivalente à celle d'un fœtus à son placenta, avec cette particularité que ce démiurge est le créateur de cette terre (comme la larve ou la chenille crée la chrysalide et donc son placenta). Lombric terrestre représente donc, chez les Miraña, aussi bien le pénis du démiurge que le cordon ombilical qui va permettre aux « enfants » issus de cette terre de vivre, comme le ferait une larve ou 
une chenille pour un imago d'insecte. Il peut ainsi être alternativement un enfant ou un pénis, un enfant ou des trompes, bref une émanation de placenta.

Dans une première approximation, la coquille de l'escargot mythique serait comparable à un cordon ombilical et à un placenta dans une matrice qui n'existe pas encore (au même titre que la chrysalide est une " poche de gestation » sans matrice). Le créateur est le fœtus qui vient prendre place sur ce placenta. La " terre » de naissance du créateur est un placenta, et le créateur est relié à ce placenta par un colimaçon. La terre n'existe donc pas encore, et cette « terre » à laquelle il est fait jusqu'ici référence n'est une terre que pour le créateur (Figure 1).

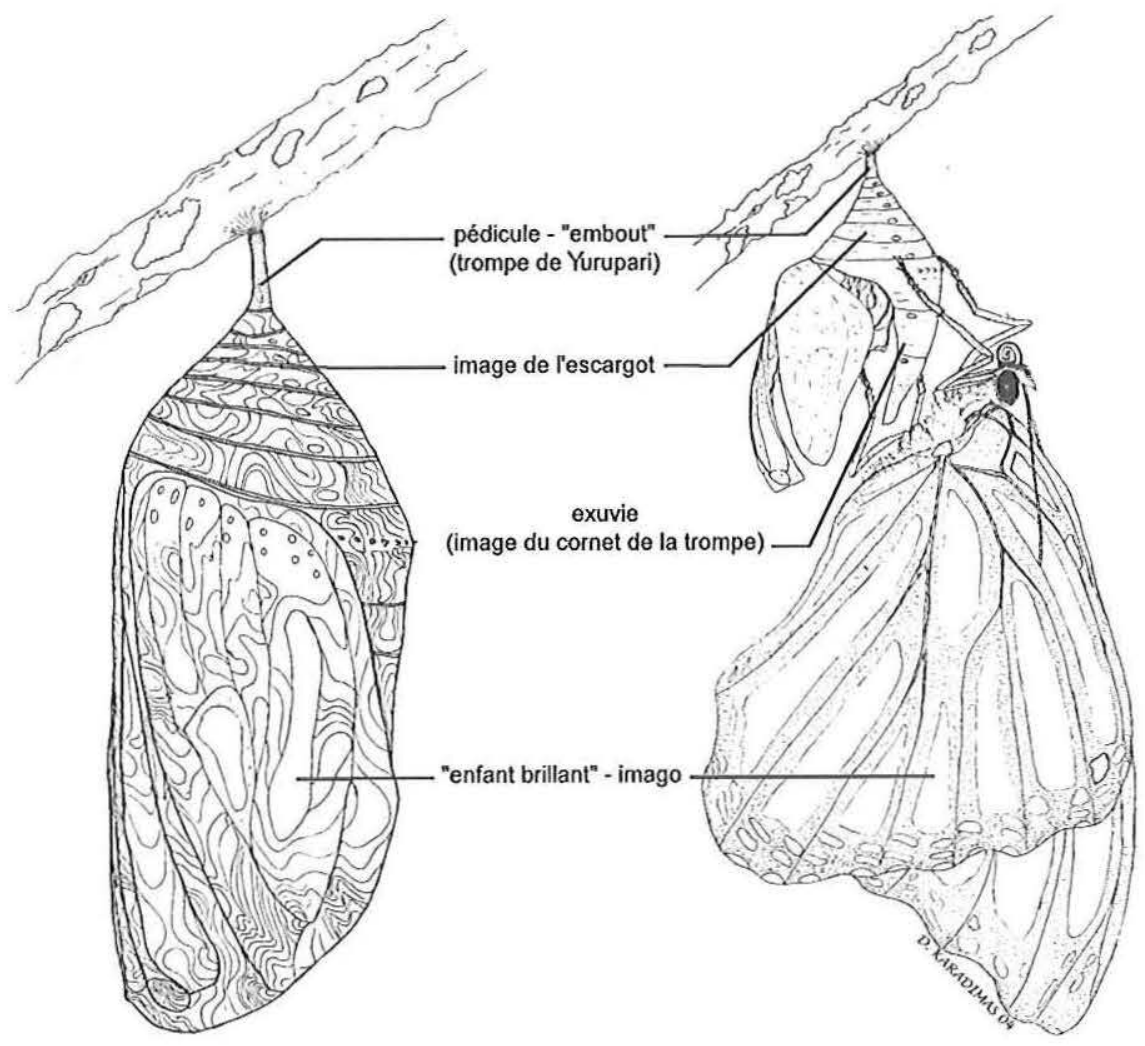

CHRYSALIDE - YURUPARI

FIG. 1 - Chrysalide de papillon comme modèle des trompes de Yurupari et de la première terre (dessin de l'auteur D. K.). 
L'escargot est une figure mythique problématique car elle inverse les éléments présents dans l'analogie faite entre l'escargot et la matrice, ainsi qu'entre la coquille de l'escargot, le lombric (cordon ombilical) et le placenta. En effet, ce qui peut être comparé à un lombric se trouve dans la coquille et forme un serpentin (les viscères de l'escargot), alors que le corps du mollusque se trouve à l'autre extrémité de ce serpentin et sort par l'ouverture de la coquille. Le corps de l'escargot est comme le fœtus relié par un serpentin à l'extrémité de la coquille en spirale qui semble tendre vers le néant. Les Miraña comparent cette coquille d'escargot à ce qui reste des mues des insectes car ils en retrouvent souvent des vides dans la forêt. En un sens, la coquille comme l'exuvie sont des restes comparables au placenta. Dans le cas de la terre du créateur, il n'y a pas encore de matrice pour ce placenta. Des associations similaires existent chez les Desana pour qui la spirale d'écorce qui forme le corps des trompes de Yurupari est associée à une odeur féminine. Les Desana utilisent pour évoquer cette écorce le terme " gahsíro » qui désigne " "l'écorce", "la coquille" et "l'utérus" " (ReichelDolmatoff 1997, p. 297). Il est donc possible de dresser provisoirement le tableau comparatif suivant :

\begin{tabular}{|l|l|l|}
\hline \multicolumn{1}{|c|}{ Escargot } & \multicolumn{1}{c|}{ Matrice } & \multicolumn{1}{c|}{ Terre du créateur } \\
\hline coquille en spirale & placenta & terre \\
\hline serpentin (dans la coquille) & cordon ombilical (lombric) & son (chant de la coquille d'escargot) \\
\hline corps du mollusque & fetus & créateur \\
\hline
\end{tabular}

Fig. 2 - Comparaison des composantes de l'escargot, de la matrice et de la « Terre du créateur ».

En les comparant avec les trompes de Yurupari, la terre du créateur, le son de la " conque » et le créateur occupent des places équivalentes à celles de la trompe, du son et de « l'enfant » que la trompe produit. L'ensemble équivaudrait à ce qui se passe dans une chrysalide. Notons que, lors de la confection des trompes, il n'y a que l'embout qui est gardé d'un rituel à l'autre ; l'écorce enroulée est jetée, comme un reste qu'elle est (c'est-à-dire équivalent à la coquille, à l'exuvie ou au placenta).

\begin{tabular}{|l|l|l|}
\hline \multicolumn{1}{|c|}{ Terre du créateur } & Chrysalide (ou nymphe des insectes) & \multicolumn{1}{c|}{ Trompe de Yurupari úíhádji } \\
\hline terre & exuvie & enroulement de l'écorce \\
\hline son (chant de l'escargot) & insecte dans la nymphe & son des trompes \\
\hline créateur & imago de l'insecte & " enfant brillant » \\
\hline
\end{tabular}

FIG. 3 - Comparaison des composantes de la « terre du créateur », de la chrysalide et de la trompe de Yurupari.

Le paradoxe est que le créateur prend place sur cette coquille, donc sur sa " terre », et qu'il semble ainsi être celui qui permet son élargissement. Tout laisse à penser que, sur le modèle des trompes, c'est le créateur qui souffle dans cette 
coquille pour l'agrandir et pouvoir se créer lui-même. Il est donc tout à la fois celui qui souffle et produit le son, comme il est ce qui est produit par le son ${ }^{5}$, c'est-à-dire l'enfant. Lorsqu'il se trouve dans cette position, le créateur est à l'extrémité placée au centre de la spirale de l'escargot qui s'enroule sur elle-même et semble n'avoir aucun commencement : elle part du néant.

Il manque cependant la matrice qui contient ces trois éléments. Avant de voir comment la création de cette matrice est présentée dans le mythe, il faut souligner une autre association que font les Miraña entre les trompes de Yurupari et la sarbacane.

\section{EMBOUT ET SPIRALE ; TROMPES ET SARBACANE}

La trompe est constituée d'un embout fait en bois imputrescible et d'une bande d'écorce souple enroulée en spirale à partir de cet embout. L'écorce est enroulée à moitié sur elle-même pour former la trompe dont le diamètre s'élargit au fur et à mesure de l'enroulement de l'écorce. Je n'ai pas vu de telles trompes et cette description m'a été faite par les Miraña. Il en existe cependant plusieurs figurations (Koch-Grünberg 1995 ; Reichel-Dolmatoff 1996 ; Hugh-Jones 1979).

Jadis, une fois le rituel terminé, l'écorce était jetée, enterrée ou brûlée, alors que l'embout était enterré dans un endroit boueux jusqu'au prochain rituel. Les Miraña expliquent qu'avant ils possédaient cinq types différents de trompes, dont trois étaient réalisées à partir de portions de tronc de palmier évidé (désignées sous le terme de rórókò " perche de ronflement»), et les deux autres à partir d'écorce enroulée (úúhádji). Les trompes en tronc de palmier sont considérées comme ñó'ñá'i (le sexe masculin, le lombric mythique, ainsi que l'enfant) et reliées au sexe masculin, alors que les secondes trompes, formées par enroulement d'écorce, sont úúhádjì et reliées au sexe féminin. Toutes peuvent être, cependant, considérées comme úúhádji puisque ce terme désigne l'interdit.

Les Miraña font également une association entre ces trompes d'écorce enroulée et les sarbacanes dont le corps est recouvert d'une écorce enroulée, mais « à l'envers ». Par cette première opposition signalée par les Miraña, retenons que la sarbacane est construite sur le même modèle que les trompes, mais en inversant toutes les valeurs des pièces de confection. La sarbacane est un équivalent du sexe masculin, alors que les trompes en écorce reproduisent une partie du sexe féminin. Par la taille et l'aspect, la sarbacane pourrait être contenue dans la trompe ; l'enroulement de l'une venant s'imbriquer dans l'enroulement de l'autre. L'union de ces deux instruments pourrait ainsi symboliser l'union entre les deux sexes. Cependant, ces deux instruments représentent davantage des versions inversées l'un de l'autre que des oppositions complémentaires.

J'ai montré comment, pour les Miraña, la sarbacane prenait modèle sur l'abdomen de certaines espèces de guêpes solitaires (Karadimas 2003). La sarbacane est censée reproduire cette forme que les Miraña voient comme le pénis de 
l'insecte. Le corps de la sarbacane reproduit l'abdomen, et la fléchette empoisonnée reproduit le dard de la guêpe. Or cet abdomen de guêpe est contenu dans une exuvie au moment de sa nymphose. Artificielle en apparence, la combinaison de la sarbacane contenue dans les trompes de Yurupari est construite sur le modèle de l'insecte contenu dans l'exuvie.

La véritable complémentarité se réalise entre les deux types de trompes. Elles forment, dans un premier temps, le couple lombric/placenta (ñóñuáiłlúúhádji) ou le couple pénis/sexe féminin. Or nous avons vu comment le «lombric » agit par succion. On peut également remarquer que les Uitoto associaient une des « flûtes » (" tube de lombric ») à une succion qui « asphyxiait » celui qui en joue. Ainsi, dans la traduction du texte de Preuss par Petersen de Piñeros et Becerra - un Uitoto - (1994) : « Kuioigo [la flûte ou la trompe] signifie littéralement "tube de lombric" ; en jouant de la flûte, on aspire le pouvoir magique du lombric kuio et la personne s'asphyxie » (ibid., p. 676, note 17).

Il n'est donc pas impossible que l'opposition entre les deux types de trompes recouvre une opposition entre deux types de « souffles »; une des trompes aspire, alors que l'autre " produit » (du son, un enfant). Les trompes ñó'ñái « aspirent », « sucent » ou tètent ceux qui en jouent ; les trompes túthádji « produisent » un souffle qui est comme l'enfant. Il m'est actuellement impossible de dire ce qui était « aspiré » par le joueur (un adulte) dans le rituel miraña et si l'initié qui portait la flûte recevait cette « succion », dupliquant ainsi la succion du sein de la mère ou du sang dans la matrice par ce même lombric. Il m'est également impossible de décrire actuellement ce que sont supposées produire les trompes en écorce enroulée lors du rituel : un nouvel être ? l'initié « transformé » ?

Pour terminer cette analyse de l'apparition de la terre du créateur sous la forme d'une coquille d'escargot, je vais présenter un « modèle réduit » de l'opposition entre trompes úúhádjì et sarbacane. Ce « modèle réduit » est un contenant de tabac réalisé dans une coquille d'escargot terrestre et un insufflateur fait dans les os d'une patte de hocco (un cracidé de l'ordre des galliformes : Nothocrax urumutum). Pour les Miraña, ils revêtent une importance particulière car ils les considèrent comme des artefacts similaires - davantage dans leur forme que dans leur fonction - aux trompes utilisées lors du rituel. Ces deux ustensiles sont utilisés chez les Miraña pour insuffler, dans les sinus, du tabac en poudre (feuilles torréfiées, pilées et mélangées à de la cendre) (Figure 4).

Le contenant à tabac est composé de la coquille d'un escargot dont le début de la spirale a été remplacé par un embout en os servant à déverser le tabac dans le creux de la main : cet embout peut être comparé à celui des trompes et au pédicule de la chrysalide; c'est d'ailleurs la raison pour laquelle l'embout des trompes est parfois mentionné comme étant un os : dans le langage utilisé par les chamanes ou par un narrateur, l'embout est un os, c'est-à-dire qu'il est comme un os dans la mesure où la narration mythique est un type d'énoncé qui oblitère la comparaison ${ }^{6}$. 


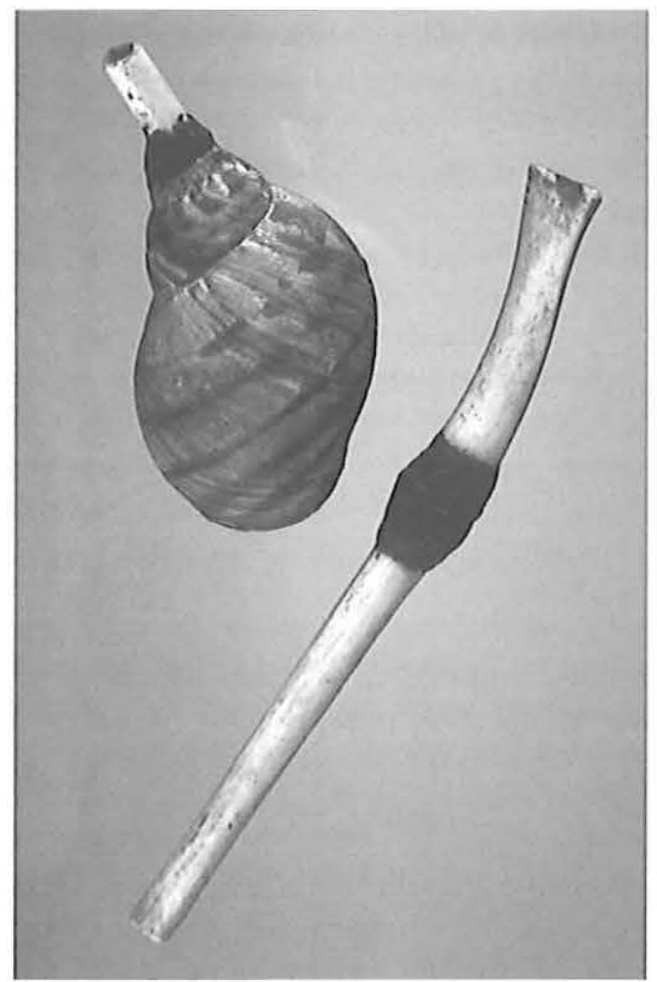

FIG. 4 - Insufflateur en os de hocco et blague à tabac en coquille d'escargot, os et verre (collection de l'auteur, D. K. Cliché de Philippe Blanchot).

La partie ouverte de la coquille a été obstruée par un morceau de miroir dépoli et calfeutré à l'aide de cire d'abeille qui sert de poix. L'insufflateur est composé d'un fémur de hocco (nimúkò) dont les deux articulations - ainsi que ceux du tibia - ont été tronquées.

Ces deux ustensiles sont comparés par les Miraña à la sarbacane, à l'escargot mythique sur lequel vient prendre place le créateur et aux trompes. Cependant, ils en inversent les valeurs puisque la coquille de l'escargot déverse de la poudre de tabac par cet embout en os, alors que la coquille d'escargot du créateur produit du son par son extrémité « ouverte ». Par cette combinaison de l'embout et de l'enroulement en spirale, le contenant à tabac est comparable à la trompe de Yurupari, tuthádji, qui est elle-même, rappelons-le, une évocation de la chrysalide. Il est donc possible de présenter ces séries d'associations : 


\begin{tabular}{|l|l|l|l|}
\hline Contenant à tabac & \multicolumn{1}{|c|}{ Trompe úúhádji } & Chrysalide ou nymphe & \multicolumn{1}{c|}{ Matrice } \\
\hline coquille d'escargot & enroulement d'écorce & exuvie & placenta \\
\hline tabac & son & insecte contenu & cordon ombilical \\
\hline embout en os & $\begin{array}{l}\text { embout en bois imputres- } \\
\text { cible }\end{array}$ & pédicule de suspension & $\begin{array}{l}\text { sperme "os " (fléchette } \\
\text { "fichée ») }\end{array}$ \\
\hline
\end{tabular}

FIG. 5 - Comparaison du contenant à tabac, de la trompe, de la chrysalide et de la matrice.

\begin{tabular}{|l|l|l|l|}
\hline \multicolumn{1}{|c|}{ Insufflateur } & \multicolumn{1}{c|}{ Sarbacane } & \multicolumn{1}{c|}{ Insecte à dard } & \multicolumn{1}{c|}{ Pénis et scrotum } \\
\hline tibia (de hocco) & corps de l'instrument & abdomen & tête du pénis \\
\hline $\begin{array}{l}\text { fémur (de hocco) (avec } \\
\text { tabac) }\end{array}$ & embout (avec fléchette) & base de l'abdomen & corps du pénis \\
\hline tabac (dans fémur) & $\begin{array}{l}\text { fléchette (engagée dans } \\
\text { l'embout) }\end{array}$ & aiguillon & sperme (dans scrotum ?) \\
\hline
\end{tabular}

FIG. 6 - Comparaison de l'insufflateur, de la sarbacane, des insectes à dard et de l'ensemble pénisscrotum.

Dans cette série d'oppositions et de complémentarités, le contenant à tabac et l'insufflateur sont des répliques inversées des deux types de trompes. En effet, si la trompe d'écorce « déverse » du son par sa partie ouverte et que cette trompe est comparée à la coquille d'un escargot, le contenant à tabac déverse du tabac à partir d'un embout qui est à la place de la partie rétrécie de la spirale (là où est placé l'embout des trompes). Entre les deux instruments, les trajets sont inversés. Les Barasana, eux, soufflent à l'aide de l'insufflateur du tabac en poudre directement dans les trous qui servent à former les notes des instruments de musique (flûtes), puis aux initiés (Hugh-Jones 1979, p. 67).

Si nous nous tournons maintenant vers l'insufflateur ${ }^{7}$, celui-ci projette du tabac dans les narines, alors que la trompe ñónái’ aspire par la bouche ou, pourrait-on dire pour être plus précis, le lombric ñóñá'i « tète » la matrice de la mère. Si lombric et pénis sont équivalents, une fois dans la matrice le lombric fait l'inverse du pénis ; en lieu et place de projeter du sperme, il tète et aspire le sang de la mère pour l'enfant. Cette opposition complémentaire se réalise également entre sarbacane et trompe ñóñá'i. Les deux instruments sont équivalents, mais n'agissent pas de la même façon, puisque l'un, la sarbacane, est un instrument létal, alors que l'autre, la trompe ñóñá'i, permet une création bien qu'elle agisse par succion.

Idéalement, la trompe ñóñáì devrait être contenue dans la trompe itúhádji puisqu'elle forme le cordon ombilical dont le placenta est matérialisé par cette seconde trompe. Les sons combinés des deux flûtes formeraient alors ce qui est transmis à l'initié.

Dans le modèle de la terre du créateur, le son qui provient du « chant de l'escargot » est ainsi soufflé par le créateur et « aspiré » par le lombric qui devrait être le serpentin dans la coquille de l'escargot. Ce chant soufflé et aspiré est donné 
à un corps qui va se former, mais qui n'est pas le créateur, puisque celui-ci « souffle » et se fait « aspirer » par le serpentin ou ñóñấi. Le corps qui se forme alors est comme un foetus, mais il est également un corps qui va devenir la terre - mais il s'agit d'une autre discussion.

Pour illustrer mon propos, j'ai reproduit, d'après une photographie du Musée de l'Or de Bogota, différents objets d'orfèvrerie précolombienne qui ont en commun, selon l'auteur de cette classification inattendue, de présenter des coquilles de gastéropodes. Ces objets proviennent de traditions et d'époques variées (Figure 7). Ils ont été réunis, sur cette photographie, de manière artificielle par un conservateur de musée et ne sont en rien l'illustration d'une classification indigène. Leur reproduction dans ce texte se justifie par le fait qu'un des objets (Figure 7, $n^{\circ} 3$ ) n'est pas une coquille de gastéropode, mais la figuration d'une chrysalide. On peut donc dire que la personne qui a procédé à cette réunion a fait une confusion. Je considère cette confusion comme un parfait exemple de ce que la logique classificatoire de l'esprit humain est capable de produire, indépendamment des cultures, dans le temps ou dans l'espace : la forme de la chrysalide évoque celle d'une coquille d'escargot, et l'artisan amérindien à l'origine de cette pièce a d'ailleurs figuré la segmentation de l'abdomen par un enroulement en spirale. On peut donc en déduire que la personne qui a choisi de mettre cet objet sur cette photographie a utilisé la même opération mentale que celle des Indiens du Nord-Ouest amazonien qui rendent la chrysalide par une image de coquille d'escargot ou par une trompe qui duplique son image grâce à l'adjonction d'un embout/pédicule à la spirale d'écorce/exuvie. Notons au passage que la figuration de la chrysalide fut un thème présent dans les sociétés précolombiennes.

À titre de comparaison, j'introduis ici la reproduction d'un dessin tiré d'un ouvrage de Koch-Grünberg (1909-19i0) et réalisé par un Indien kaua de langue arawak du haut Rio Negro (Figure 8). Dans ce dessin, à côté des masques figurant des papillons et des têtes de scarabées pendant un rituel, apparaissent des chenilles et des chrysalides. Le traitement graphique de la « segmentation » des formes larvaires des hyménoptères dans les costumes-masques (Figure 8e et i), par le dessinateur indigène, doit être mis en parallèle avec les constatations antérieures.

Enfin, ajoutons à cette association entre le modèle des trompes de Yurupari et celui des coquilles de mollusques avec un tube creux, le cas issu de la mythologie jivaro qui inverse le rapport entre embout et cornet :

Uñushi [le paresseux], qui s'était égaré, apprit enfin son infortune. Il accusa, on ne sait pourquoi, sa mère la Lune d'avoir aidé les coupables, la battit et l'enfouit dans un trou. Engoulevent, soupirant éconduit de Lune, arriva sur les lieux. Conseillé par Colombe, il fabriqua une trompe de coquillage, s'introduisit dans le tronc creux d'un palmier qui gisait là et sonna de son instrument. À cet appel Lune jaillit hors du trou, traversa le tronc creux comme un dard de sarbacane en chassant engoulevent devant elle et fila tout droit jusqu'au ciel. (Lévi-Strauss 1985, p. 99) 

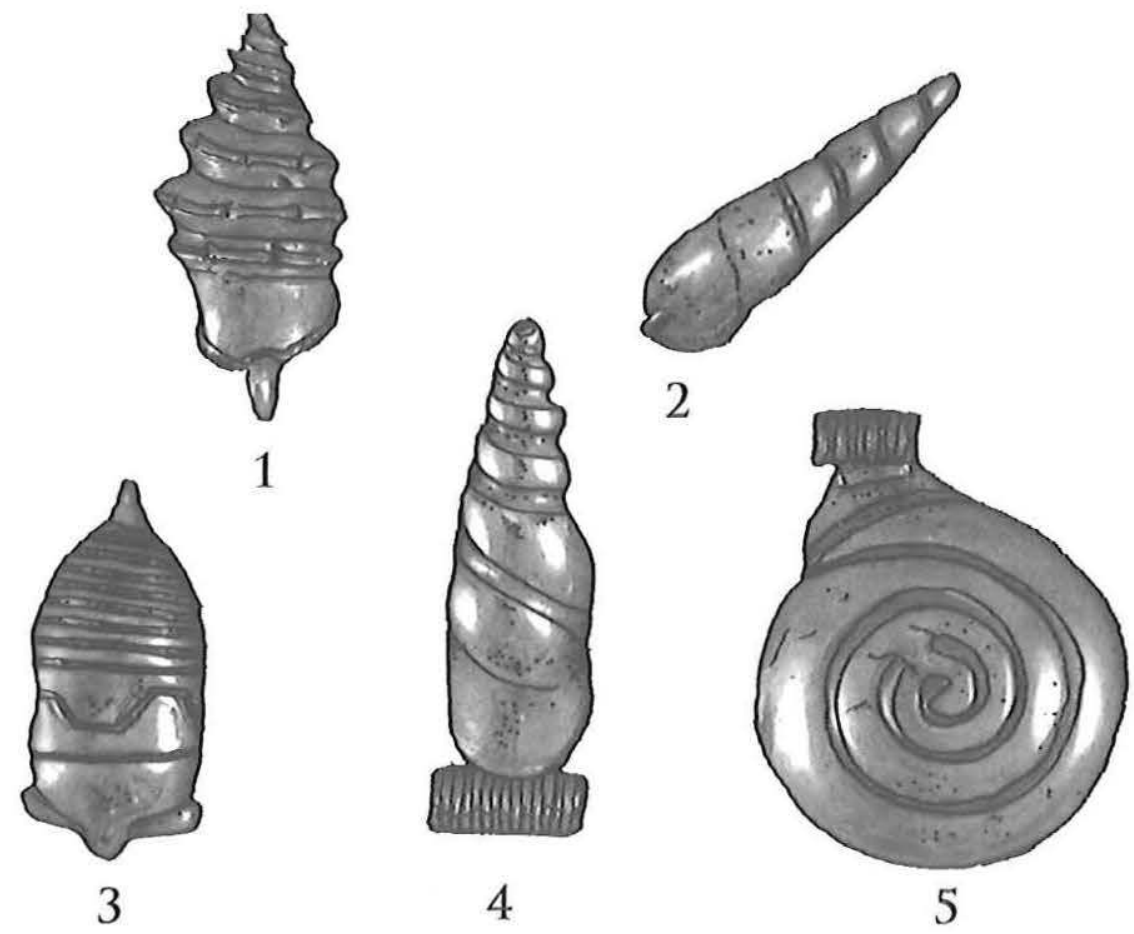

Fig. 7 - Réunion de différentes « spirales » de l'orfèvrerie précolombienne ; l'objet $n^{\circ} 3$ est une chrysalide (dessin de l'auteur, D. K., réalisé à partir d'un cliché de Carrieri pour le Musée de l'Or de Bogotá, in Hemming 1984, p. 197).

On remarquera d'emblée que les éléments qui composent les trompes du Yurupari dans le Nord-Ouest amazonien se trouvent ici inversés, à l'écorce enroulée en spirale près, remplacée dans ce mythe jivaro par une conque. Ainsi, au lieu du modèle tube-embout/spirale enroulée des trompes de Yurupari, c'est la conque de coquillage qui est contenue dans le tube formé par le palmier creux : ce tronc sert en quelque sorte de cornet et la coquille d'embout. L'inversion est complète car le musicien est contenu dans le tube et souffle à l'intérieur de celui-ci ; Lune, jaillissant du trou, traverse ce tronc creux en chassant devant elle son occupant (c'est-à-dire qu'elle suit un chemin inverse de celui qu'emprunterait le son produit par le joueur). Comme le note Claude Lévi-Strauss (1985), l'instrument de musique fait ici office de sarbacane, et le souffleur, Engoulevent, est relégué dans le rôle de la fléchette. Dans cette variante combinatoire jivaro des instruments de Yurupari, c'est donc bien le modèle de la conque qui est premier.

À titre spéculatif, on pourrait faire remarquer que l'utilisation systématique de conques à des fins rituelles se retrouve dans les Andes péruviennes avec l'utilisation des pututo ou potuto (conque de gastéropode marin agrémentée, ou 


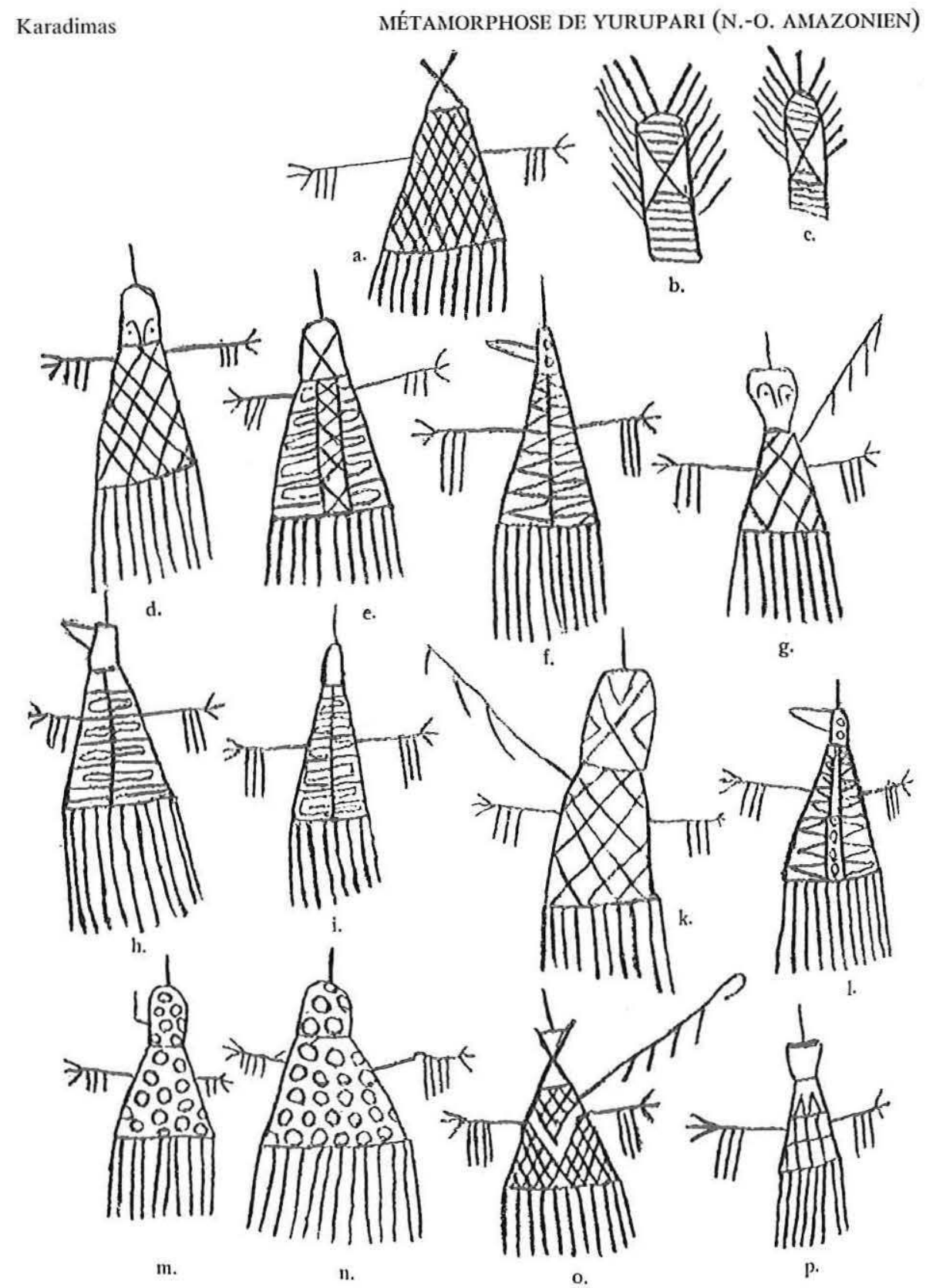

FIG. 8 - Dessin tiré du recueil de Koch-Grünberg exécuté par un Indien Kaua (arawak) figurant des costumes-masques. Parmi les papillons $(f, h, l)$ et les têtes de scarabées $(b, c)$, on notera le traitement graphique donné aux chrysalides et chenilles avec leur segmentation (e, i) (d'après Koch-Grünberg 1909-1910, figure 118). 
non, d'un embout). Ces conques produiraient un son qui aurait, selon une tradition andine contemporaine, permis à l'empereur Pachacutec de renaitre ; ces trompes serviraient là encore comme instruments de renaissance. Il faut noter que, dans les basses terres, d'autres formes de trompes ont été désignées par un terme similaire : "According to Humboldt [...], the Indians of the Atabapo and Inirida area (north of Vaupès) used trumpets made of clay, called botuto [...] " (Hugh-Jones 1979, p. 137).

\section{COMPARAISON AVEC LES DONNÉES DES GROUPES DU VAUPÈS}

Les données sur les groupes de langue Tukano et Arawak du Vaupès sont essentiellement tirées d'un ouvrage de Reichel-Dolmatoff $(1996)^{8}$. Ce livre présente une analyse de quatre textes (numérotés en chiffre romain de I à IV) qui sont des narrations ayant en commun de décrire l'origine des flûtes et leur passage d'une appartenance féminine à une possession masculine. Ces textes accréditent aussi, aux yeux des personnes qui les énoncent, l'origine de la sexualité et des interdits qui lui sont liés.

La trame commune des quatre textes, que je synthétise à l'extrême, est la suivante :

Une guêpe de couleur métallique [IV] est brûlée et ses cendres donnent naissance au palmier paxiuba (Socratea exorrhiza) grâce auquel on fait les flûtes et les embouts des trompes. Un père avait préparé quelque chose pour que son fils apprenne. Les sœurs, ayant entendu parler de ce quelque chose, partent le voir au débarcadère (là où l'on se baigne d'habitude). Elles l'aperçoivent sur (au pied d') un palmier paxiuba (en fleur) : ce quelque chose - Tonnerre [I], un ancêtre [II], - miria pora [III], la racine [IV] - se balançait d'avant en arrière au gré du vent [I], pénétrait [IV]. Ce mouvement, ou cet état des choses (image de la pénétration), éveille, chez ces sœurs, un désir. Elles remontent voir leur père afin qu'il leur prépare un "bâton à manioc » [I], elles soufflent dans les flûtes [II]. Le père comprend qu'elles ont vu ce qui était destiné à son fils. Il envoie ses aides, des rongeurs, afin qu'ils sectionnent le palmier. Mais ces derniers ne voulaient pas révéler le secret de l'attraction (produit par le " bâtonnet de la vulve ", c'est-à-dire le clitoris). Le premier, le grand écureuil, monte dans la " demeure des hommes », puis chacun en fait autant. C'est pour cela, affirme le narrateur, qu'il y en a de différentes tailles (en parlant des sections de palmier, des membres masculins et, selon Reichel-Dolmatoff (1996), des clitoris). En faisant cela, il crée les différents clans (groupes de filiations), chacun avec une section de palmier (prélevée sur un seul tronc). Le père, lui, ne restera qu'avec une petite section du palmier qui est l'embout des trompes du rituel. De là-haut, il arrangea le morceau afin de berner les femmes. En voyant arriver un si petit morceau, les sœurs le refusent de façon agressive en réclamant quelque chose de plus grand. 
Le père prépare ce quelque chose et le dépose auprès du débarcadère pour qu'au petit matin, son fils se l'approprie. Celui-ci se fait pourtant précéder par ses sœurs qui volent l'embout, se baignent et cherchent où le placer sur leur propre personne. En fait, elles le placent comme les racines du palmier [I, \$127], puis s'enfuient pour créer leur propre maison communautaire. À partir de là, elles deviennent déviantes (découvrent l'homosexualité féminine). Dans le texte I, elles abandonnent l'embout des flûtes à cause de l'endroit où elles l'avaient placé, soit dans leur entrejambe. L'esprit de l'embout, un petit rongeur (un acouchi, genre de petit agouti : Myoprocta pratti), s'enfuit effrayé lorsqu'elles s'enfoncent l'embout dans leur entrejambe [II].

Les hommes doivent empêcher que l'acouchi puisse retourner dans le tube (l'embout). À cet effet, les hommes créent la mante religieuse (textes II, III et IV). L'insecte sectionne l'embout et prend sa place dans l'entrejambe des femmes, non sans que les hommes aient préalablement transpercé ou creusé la mante religieuse [II]. Dans le texte III, la mante ne fait que fendre la terre avec sa patte prédatrice (au lieu de trancher l'embout). Dans le texte I, les hommes retrouvent les femmes qui ont abandonné l'embout et se proposent de les inséminer : du haut de la maloca, ils mâchent du piment afin de saliver abondement [IV] et afin de descendre le long de ce fil [I]. Chacun s'y essaie, mais seul le plus jeune y parvient [III]. Il les insémine, mais ses frères le poussent et le tuent. Ces derniers inventent les formules pour le ressusciter : la reproduction. La reproduction se fera comme une mue : c'est comme cela qu'il renaîtra [I, §155].

Dans le texte II, après que la mante ait sectionné les embouts, les femmes tentent de s'enfuir de la maison communautaire maintenant investie par les hommes ; certaines récupèrent les embouts et les replacent entre leurs jambes. Dans les textes III et IV, les femmes se doivent de cacher les embouts ; certaines les cachent dans leur entrejambe, les autres aux alentours et permettent aux hommes de les récupérer. Dans le texte II, d'autres femmes s'en détournent et laissent les embouts, elles s'enfuient en forêt ; les hommes les récupèrent pour eux, mais ces embouts n'avaient plus de force (l'acouchi est en fait l'esprit, la vigueur, de l'embout). Chez toutes ces femmes qui les ont placés dans leur entrejambe, les embouts restent comme clitoris : il est l'organe qui refuse de céder et les empêche d'avoir des enfants ou d'accoucher.

Dans le texte I, une fois le mode de reproduction (par changement de peau) rétabli, les femmes refusent de bien recevoir les hommes dans leurs malocas. Malgré cela, les hommes les investissent et, ce faisant, déchirent l'écran fait d'une natte de palmier (évocation de la défloraison ?). Les femmes s'enfuient de nouveau et trouvent refuge plus bas sur le fleuve. Les hommes arrivent et prennent contact avec "celui qui accompagne les femmes », un allié. Ce dernier leur propose de se multiplier comme la grenouille pipa pipa et les opossums (c'est-àdire sans gestation). Les hommes refusent et revendiquent le fait d'être les enfants des hommes ; les femmes doivent porter leurs enfants. La reproduction, aupara- 
vant instituée, se fera par le sexe des femmes lorsqu'elles auront leur " eau ». Reichel-Dolmatoff évoque l'urine : selon la physiologie miraña, il s'agirait du liquide vaginal. En faisant cela, les hommes commencent à se disperser et à créer les différents groupes. En récupérant les embouts, les hommes laissent la séduction aux femmes, mais récupèrent la parole [I et IV]. Les femmes, pourtant, « ayant, les jours de grands désirs sexuels, placé leur main dans "l'obscurité du palmier", ont senti l'essence de l'embout » et les hommes ont placé le quartz dans cet entrejambe. Les femmes pensaient pouvoir tirer profit de cette acquisition, mais les hommes ne se laissèrent plus séduire. Ils étaient plus forts (Figure 9).

\section{Phallus et Clitoris : UN MÊME « BOIS »}

L'ensemble des narrations des quatre textes tourne autour de la récupération des éléments pris sur le palmier par les femmes, puis placés entre leurs jambes, et des actions entreprises par les hommes pour se les approprier.

D'après Reichel-Dolmatoff (ibid., p. 165), il semble que les différents termes utilisés pour désigner Yurupari dans les groupes Tukano renvoient à des images évoquant le clitoris. Il apparaît que ce que Yurupari et les trompes représentent c'est en fait la matière ou le « bois » qui, d'un côté, va créer le clitoris, puis, de l'autre, le pénis, lorsque les hommes auront récupéré ce qui leur appartient.

La narration souligne, en tout cas, le fait que l'embout des flûtes représente, alternativement, clitoris et pénis. En d'autres termes, on peut considérer qu’il existe dans cette tradition une reconnaissance du fait que l'organe de plaisir des deux sexes possède une origine commune. " Yurupari » est donc présent dans les deux sexes, avec cette précision qu'il était d'abord chez les femmes, puis il a été récupéré par les hommes qui l'ont ôté de l'entrejambe des femmes. Ces éléments pourraient laisser penser que, dans sa composante sexuelle, « l'affaire » de Yurupari s'articule autour de l'existence de deux organes perçus comme équivalents, mais ayant une expression réduite chez les femmes et développée chez les hommes.

Revenons sur certains aspects de la narration, notamment le petit acouchi qui va être récupéré par les hommes et qui représente la vigueur de leur membre.

La phrase suivante : "When they introduced it by pushing it in, he went out. // He went out? // Yes, the one they call cutia. // Yes " (Reichel-Dolmatoff ibid., p. 188, II, §59) évoque l'introduction de l'embout de bois de palmier, en tant qu'objet sexuel, par les femmes qui s'étaient enivrées (ibid., p. 187, II, §57). Il est précisé « qu'il est sorti ». Celui qui est désigné ici est l'acouchi associé au clitoris (Reichel-Dolmatoff le dit explictement : «Cutia symbolizes the clitoris »), dans la relation qu'il entretient avec le tipiti, ou presse à manioc, qui lui sert de gîte dans la maison communautaire. Également perçu comme gaine, le tipiti est le plus communément associé à l'image du conduit vaginal, mais aussi d'un contenant 

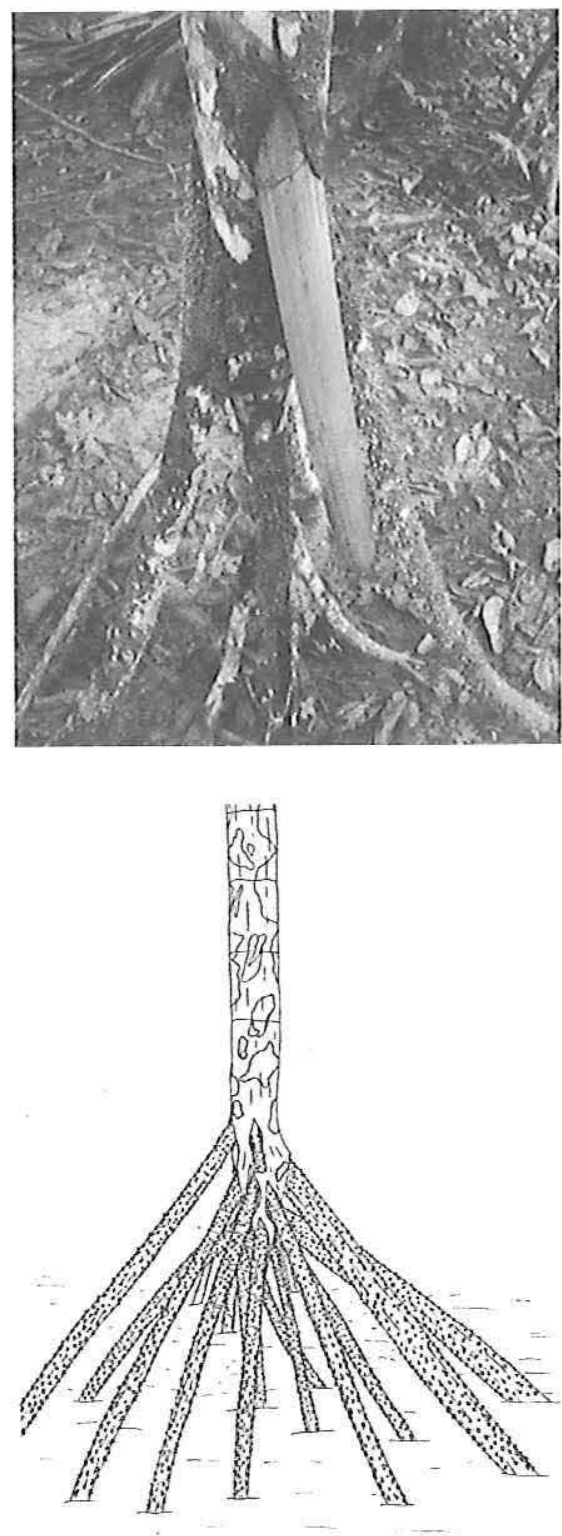

FIG. 9a et b-Racines échasses émergeant du tronc du palmier paxiuba par des fentes verticales et ovales; dans la mythologie de Yurupari, ces fentes évoquent, en association avec la racine - image du phallus -, l'androgynie primordiale à laquelle le complexe de Yurupari met fin par sectionnement, créant ainsi la sexualité (je remercie Stephen Hugh-Jones pour la photographie qui est sienne; le dessin est de l'auteur D. K.). 
permettant une transformation ${ }^{9}$. L'image suggérée est celle de la relation entre le petit rongeur - le clitoris - et le tube ou la gaine de vannerie - le capuchon clitoridien. Il semble que l'analogie entre le clitoris et le rongeur découle du fait que le clitoris « sort » de son « refuge » ou de son « gîte » comme le ferait le petit rongeur, animal domestiqué qui vit dans la presse à manioc. Cette dernière n'est pas ici utilisée comme image du vagin, mais comme celle du capuchon clitoridien. La correspondance se fait donc sur trois niveaux: clitoris/capuchon, pénis/prépuce et gaine vaginale/enfant à naître.

Nous verrons plus avant comment cette double image, apparemment paradoxale puisqu'un même objet peut à la fois être gaine clitoridienne et gaine vaginale, n'implique pas, de fait, d'opposition mutuelle entre ces images (complémentarité entre clitoris et l'enfant qui naît...).

Dans le texte II, $\$ 60$ (ibid., p. 188), le rongeur saute de la vannerie sur le sol et les hommes se demandent ce qu'ils doivent faire afin de l'éloigner de sa retraite et le récupérer ("What shall we do in order to take it away? ?). Dans les textes II, III et IV qui sont desana (le premier étant d'origine tukano), il est dit : " This is what they were saying when thinking it over; and thus they institutionalized the mantis./I They institutionalized it ?/I Yes ». Ce passage (ibid., p. 188, II, §62, sq.) est relativement énigmatique puisqu'il propose "d'institutionnaliser la mante religieuse » afin de récupérer cet élément pour les hommes. Un des problèmes réside dans le terme bari sero qui désigne, selon Reichel-Dolmatoff, la mante religieuse et est utilisé comme synonyme de $\sim$ poreró, terme qui fait référence à l'embout des trompes de Yurupari [I, §153]. Pour Reichel-Dolmatoff, Yurupari et bari sero sont synonymes. Attardons-nous sur les qualités retenues par les Tukano concernant l'insecte en question ${ }^{10}$.

Reichel-Dolmatoff aborde le comportement particulier de la femelle de la mante lors de la fécondation : celle-ci dévore le mâle qui, bien qu'à moitié grignoté à partir de la tête, continue de produire des mouvements de l'abdomen afin de poursuivre la fécondation de la femelle. L'étymologie même du terme bari sero évoquerait aussi cet aspect, mais d'une façon singulière : «bari sero : "grappin dévoreur"'/"fourche dévoreuse", d'après / serol : fourche ou grappin pour décrocher les racèmes ou les fruits des grands arbres » (ibid., p. 165 sq., notes et commentaires du texte II). De mon point de vue, l'étymologie serait fondée sur une analogie entre les membres antérieurs de la mante religieuse qui sont ravisseurs et bari « dévorer, manger de la viande » (Ramirez 1997). Le terme bari sero serait ainsi à comprendre comme "grappin ravisseur ", dans la mesure où la mante se sert de ses membres comme de grappins (Reichel-Dolmatoff 1996, p. 225, III). Ce grappin, instrument grâce auquel la mante est désignée chez les Tukano, existe aussi chez les Miraña et sert à décrocher les racèmes et autres fruits haut perchés dans les arbres ou les palmiers. Il est formé par une longue perche à l'extrémité de laquelle est fixé un bâton plus court grâce à une liane. L'ensemble forme un angle obtus similaire en tous points aux membres ravisseurs de la mante 
religieuse. Lorsque cet instrument est utilisé, le pédoncule- ou tout autre élément qui maintient le fruit ou le racème à l'arbre - est passé dans le grappin qui, plus qu'il ne le sectionne, l'arrache de son support ; ce qui est fait avec le pénis/racine dans le mythe.

Toutefois, Reichel-Dolmatoff (ibid.) considère que la « fourche » ainsi désignée est celle de l'entrejambe féminin. Dans les commentaires qu'il propose sur les images censées générer le terme bari sero, il fait remarquer : " The image which are consistently referred to when discussing bari sero are a point (A) and a cut (V); the Indians compare the former to a clitoris, to the pointed mouth of the mantis, to a small sprout on a stem, or to a distant maloca or a hill on the horizon [...]" ; il souligne : "These are not free associations elicited by the ethnologist but are causal comparisons made in conversation which, nevertheless, represent recurrent images » (ibid., p. 167). En s'appuyant sur l'utilisation d'analogies formelles, marquées par un classificateur nominal (vi/objet conique), cet auteur nous fait comprendre que le clitoris est placé dans un objet conique (clitoris et prépuce), bien que son rapprochement, fondé sur une homophonie avec vi'i (maison), soit à rejeter. Bref, si la tête de la mante religieuse doit jouer le rôle de l'image du clitoris (ibid., p. 189, II, §65) et si le terme bari sero est utilisé comme synonyme des trompes, c'est qu'il existe une relation de complémentarité dans laquelle l'écorce des trompes joue le rôle du prépuce clitoridien, dans la mesure où l'embout de ces trompes est le clitoris.

Après avoir présenté les attributs des différentes flûtes, le texte continue avec la description de $\sim$ poreró, l'embout de la trompe de Yurupari fait en bois de paxiuba. Le narrateur fait remarquer que : "He too has many adornments, the $\sim$ poreró. /I The poreró ? Yes.// Yes », puis dans les commentaires de Reichel-Domatoff (ibid., p. 87, I, \$160) : "Buhsátiri : "from buhsátise/to be adorned. This refers to persons 'armed' with powers, i.e., people who wear feather headdresses, people who where born still enclosed in a caul" ". Il s'agit de ceux qui sont dits " nés-coiffés » avec une partie de la membrane amniotique recouvrant leur visage, ou leur servant de "coiffe»" ${ }^{11}$. S’il existe une série d'équivalences entre la membrane amniotique et l'enfant à naître, d'un côté, et le capuchon clitoridien et le clitoris, de l'autre - l'enfant qui naît coiffé est similaire au clitoris et à son capuchon clitoridien qui est un élément conique équivalent aux trompes de Yurupari -, cela revient à dire qu'il existe une équivalence entre la « coiffe », la membrane amniotique et les trompes de Yurupari (en l'occurrence l'écorce enroulée en spirale). En d'autres termes, le " pouvoir » ornemental des trompes est équivalent au pouvoir acquis par un enfant né-coiffé, l'écorce enroulée en spirale des trompes étant alors équivalente à la membrane amniotique.

Or la mante religieuse est censée se balancer d'avant en arrière au rythme du vent [I, §13]. Une des espèces existant en Amazonie (Choeradodis rhombicolis), dite aussi "mante à capuche" (hooded mantis en anglais, Figure 10), a une attitude mimétique et défensive qui consiste à imiter le feuillage. Cette mante 


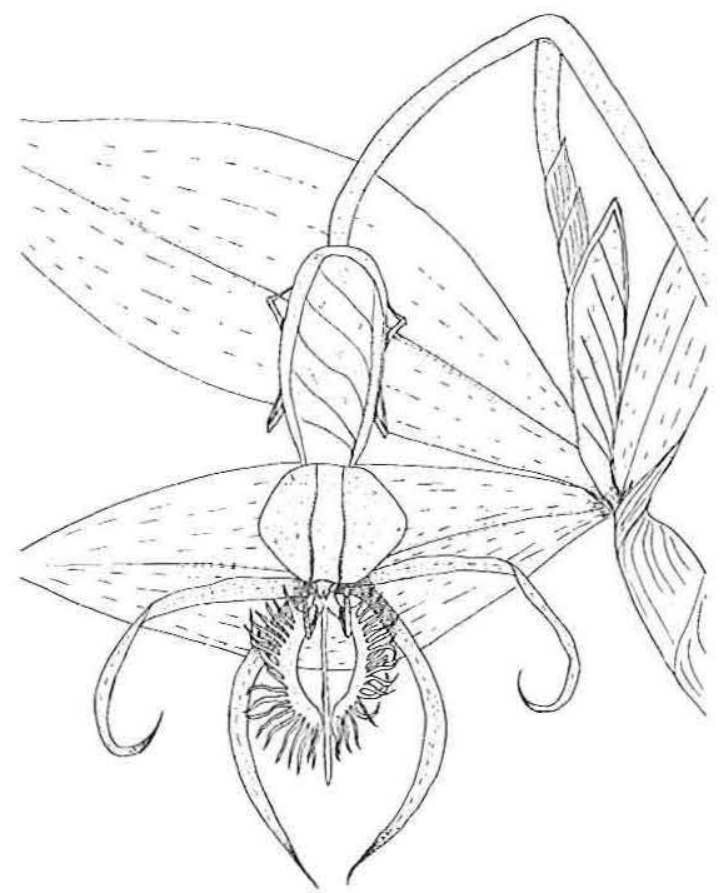

FIG. 10 - Choeradodis rhombicolis à l'affût sur l'orchidée amazonienne Epidendrum ciliare: pour se camoufler, la mante sectionne à l'aide de ses membres inférieurs le gynostème ou " colonne » et prend sa place sur la fleur. En association avec l'image de la fleur comme sexe féminin, c'est ce comportement ainsi que son pronotum étiré, image du clitoris et de son capuchon, qui rendent compte de la présence de l'insecte dans la narration (dessin de l'auteur, D. K.).

produit spontanément un type de balancement caractéristique laissant penser que cette « feuille » bouge sous l'effet du vent. Cette attitude de balancement, une fois anthropomorphisée, peut être perçue comme une simulation d'un va-et-vient identifiable à des mouvements de types copulatoires.

De part la présence de cette « capuche », il semble bien que ce soit cette espèce de mante qui soit ici désignée : il n'y a que le bout de sa tête qui dépasse de sa capuche, le reste de la partie centrale du pronotum correspondant à la hampe du clitoris.

Il convient d'ajouter que, dans la mythologie des groupes tukano, l'équipollence entre clitoris et nouveau-né passe par un traitement similaire de leur présence au sein des flûtes. Il existe un être dans les deux cas. Dans le cas du nouveau-né, le sac amniotique sert de « coiffe » au fœtus lorsqu'il naît coiffé, c'est ce qui apparaît dans le modèle de la chrysalide chez les Miraña. Dans la mythologie tukano, cet être est représenté par le clitoris, lui aussi « coiffé » et personnifié par la mante à capuche ${ }^{12}$. 
Pour autant, les quatre textes que présente Reichel-Dolmatoff ne sont pas exempts de références au modèle de la chrysalide. Il semble juste que, là où j'insiste sur l'équivalence entre chrysalide/fœtus/trompes pour la mythologie miraña, Reichel-Dolmatoff insiste sur les équivalences entre mante religieuse/clitoris/trompes dans la mythologie tukano. Les deux modèles peuvent toutefois être compris comme un seul à deux valeurs complémentaires. La référence clitoridienne, sorte de trace persistante de l'ancienne présence de phallus féminin pour la mythologie tukano, n'est pas soulignée dans mon analyse des trompes de Yurupari chez les Miraña. De même, les références au fœtus, et donc à l'association avec le modèle de la chrysalide, bien que présentes dans les textes cités, n'ont pas été développées par Reichel-Dolmatoff. Il convient, dès lors, de remarquer que c'est la combinaison structurale suivante qui rend compte, au mieux, de cette complémentarité :

capuchon : clitoris :: coiffe (poche amniotique/placenta) : nouveau-né :: cornet en écorce : embout des trompes :: cape : mante religieuse :: exuvie : imago

Parallèlement à ce constat, l'ensemble des descriptions contenues dans ces textes montre que la préoccupation première du mythe est une castration primordiale s'effectuant sur les femmes qui ont récupéré le phallus. La « récupération » des trompes qui suit est ainsi équivalente à une castration. Or le troisième texte donne assez clairement une indication sur le fait qu'il ne s'agit pas seulement d'une castration, mais aussi d'une excision. La mante religieuse semble être aussi mentionnée dans la narration pour ses « avant-bras », utilisés comme instrument de prédation à la façon de pinces dans lesquelles elle maintient ses proies. Si cet instrument est évoqué dans le cours de la narration, c'est que la castration des femmes qui suit se fait à l'aide des membres ravisseurs de l'insecte, fonctionnant comme des pinces ou comme un grappin. L’image ainsi générée par la narration est celle d'un instrument pouvant sectionner le membre viril encore présent sur les femmes. Cet acte aura pour conséquence que la mante religieuse prendra la place du membre viril : vue de face, la tête de l'orthoptère occupe la place du clitoris, sa " capuche », celle du capuchon clitoridien. Les trois énoncés suivants rendent compte de ce parallèle :

If he had pushed them with the mantis, the women would have been used up. "But instead of pushing and touching the woman, he pushed and touched the earth. This is why a deep cleft opened in the earth. If he had pushed and touched [them], the women would have been finished ». (Reichel-Dolmatoff ibid., p. 226, III, \$21)

En d'autres termes, si les femmes avaient été atteintes par cet instrument, elles auraient été « finies », parachevées, c'est-à-dire, selon les textes, soit entièrement castrées, soit entièrement déflorées. Bref, elles échappent ici, d'après la première interprétation, à l'excision et, d'après la seconde, à la défloration. Le texte 
précédent présentait ainsi le fait de «creuser » une cavité pour y loger la mante religieuse - incarnant là le clitoris -, alors que l'insecte est ici l'instrument qui aurait permis de parfaire les femmes, c'est-à-dire de les rendre entièrement complémentaires aux hommes en ne leur laissant aucune trace de cette proto-virilité.

La suite du mythe ou de la narration décrit la fuite des femmes emportant cette « chose » avec elles. Certaines décident de la cacher en dehors de leur corps, permettant ainsi aux hommes de la leur prendre, alors que d'autres la cachent en elles, entre leurs jambes [III, \$29]. De cette façon, elles gardent une certaine ambiguïté dans leur corps et dans leur sexualité.

Une des questions que génère le rituel de Yurupari est de savoir pourquoi les flûtes « sacrées » sont interdites à la vue des femmes. Dans un second temps, la question adjacente à la première est de savoir pourquoi la sanction devrait être la mort ou le viol. Si l'on prend comme point de départ de la réflexion le fait que les trompes représentent l'organe de reproduction féminin avec le fœtus (c'est-à-dire l'ensemble utérus/fœetus/placenta et cordon ombilical), il ne devrait pas y avoir d'obstacle culturel à ce que les femmes puissent prendre connaissance de ces instruments (puisqu'elles auront de facto à faire face au moins au placenta lors de l'accouchement). En revanche, si l'on prend en compte la seconde valence accordée aux trompes lors du rituel du Yurupari, à savoir celle du clitoris, les raisons culturelles qui motiveraient une telle interdiction sont plus présentes. En prenant comme élément de motivation le type de sexualité lié à cet organe, il faut reconnaître que les groupes du Nord-Ouest amazonien lui attribuent une nature " masculine " : une fois récupérée, la matière qui, dans le mythe, a servi à la confection des flûtes, formera les phallus des hommes. En d'autres termes, connaître la fonction et l'origine des flûtes, c'est connaître la fonction du clitoris dans la sexualité féminine et, en conséquence, savoir que cet organe est pensé comme étant placé du côté du masculin. Énoncée de la sorte, cette proposition revient à dévoiler qu'il existe une ambiguïté sexuelle liée à la connaissance de cet organe, mais également à sa manipulation. Or, dans une partie de la narration recueillie par Reichel-Dolmatoff, ses informateurs insistent pour souligner que, lorsque les femmes étaient en possession des flûtes/phallus, elles s'étaient retirées ensemble dans une maison communautaire et utilisaient entre elles les flûtes en tant qu'instrument sexuel, laissant penser à un comportement homosexuel. En ce sens, l'interdiction de la vision des flûtes relèverait d'une injonction d'hétérosexualité, c'est-à-dire d'une obligation de subir une fécondation intra-utéro.

L'application de la peine de mort pour les femmes ayant vu des flûtes, aujourd'hui bien plus une menace qu'une réalité, relèverait de l'interdiction d'une forme de sexualité liée au clitoris et assimilée à une pratique homosexuelle. La pratique du viol en substitution au meurtre est en ce sens symptomatique : elle est ici à comprendre comme une imposition forcée de cette obligation d'hétérosexualité. De même, le viol signe l'équivalence entre hétérosexualité et relation 
prédateur/proie telle qu'elle est perçue par les hommes ${ }^{13}$. Le viol est donc à comprendre comme un acte létal vis-à-vis de la composante masculine qui se révèle chez les femmes avec la connaissance de l'organe de plaisir assimilé à celui d'un homme. En réintroduisant la présence, dans le discours, du clitoris, et en en faisant un équivalent du pénis, les groupes Tukano soulignent que la différence des sexes n'est pas juste liée à l'activité sexuelle, mais aussi et surtout à la différence des modes de reproduction.

Pour que cette fécondation puisse avoir lieu, il faut, dans le rituel, rendre les hommes aptes à « inséminer ». C'est vers cet aspect de la narration et du rituel qu'il faut maintenant nous tourner.

\section{UNE REPRODUCTION SANS PLACENTA}

Dans le commentaire fait à propos des modèles donnés pour comprendre de quelle façon doit se faire la reproduction (Reichel-Dolmatoff ibid., pp. 12, 96, I, \$196), les deux animaux pris comme modèle sont l'anoure pipa pipa et la sarigue. Ces deux espèces sont emblématiques par leur mode de gestation : la grenouille incube ses œufs dans des cavités dorsales (c'est-à-dire qu'il n'y a pas de gestation intra-utérine) ; la sarigue, animal marsupial, ne « porte » pas sa progéniture dans une cavité utérine, mais à l'extérieur, dans une poche réservée à cet effet où le petit, qui ne mesure à la naissance que quelques millimètres de longueur et qui ressemble plus à une larve qu'à un mammifère, reste accroché à un téton grâce auquel il se nourrit jusqu'à maturité. Dans l'un comme dans l'autre cas, il s'agit d'une gestation extra-utérine qui, dans le cas de la sarigue, n'implique pas la formation d'un placenta, c'est-à-dire la création d'un double de la personne qui s'est nourri du sang de la mère. Les alliés, dans le texte I, refusent ce mode de reproduction et affirment au contraire que les femmes doivent accoucher.

Vient ensuite l'affirmation que les hommes devraient être des enfants des hommes : "we shall be the sons of men " (Reichel-Dolmatoff ibid., pp. 12, 97, I, $\S 198)$, c'est-à-dire idéalement entièrement conçus par les hommes. Pour que ceux-ci puissent construire un rituel dans lequel ils créent une gestation extrautérine grâce aux trompes de Yurupari, il faut que les femmes se voient imposer une gestation intra-utérine. La récupération des flûtes par les hommes est donc, dans le discours, une justification de la dissymétrie des sexes face à la reproduction humaine. Ce que les femmes ont perdu avec les flûtes est cette faculté de produire de l'humain en dehors de leur corps. Ce que les hommes se sont accaparé est non seulement cette faculté de produire en dehors de l'utérus (c'est ce qu'ils affirment en utilisant des modèles, dans le règne animal, qui possèdent cette faculté), mais aussi la liberté de restreindre cette production aux seuls individus du sexe masculin (rite d'initiation masculine). Les femmes, quant à elles, sont obligées de donner le jour aux deux sexes. Le rite d'initiation de Yurupari justifie 
la réclusion liée à l'avènement des premières règles, non pas sur le seul rapport au sang, mais sur ce que ce sang produira : l'attraction d'une âme et sa matérialisation sous forme de placenta, chez les Miraña pour le moins.

\section{REPRODUCTION, GESTATION INTRA-UTÉRINE, MUES ET MÉTAMORPHOSE DES INSECTES}

Pour aller plus loin, disons que si les trompes de Yurupari sont évoquées comme un équivalent de l'accession à la faculté de reproduction sur le modèle des saignements menstruels ou des « mues internes », c'est-à-dire des changements de peaux induisant un saignement, il faudrait que les hommes subissent un saignement équivalent. Or une des phases de l'initiation masculine est justement de faire subir aux adolescents une série d'épreuves durant lesquelles ils recevront des coups de fouet dont le but premier est, semble-t-il, de leur faire passer une étape dans la croissance corporelle ; celle-ci ne peut se faire, apparemment, qu'accompagnée d'un saignement. Cette «mue» à laquelle sont contraints les jeunes devrait être comparée à l'image des chenilles/larves qui subissent plusieurs changements de peaux avant d'aborder le processus de la métamorphose. En ce sens, les coups de fouet et le fait de voir les trompes ne sont pas des équivalents répétitifs de la même action rituelle : ces actions reprennent les évolutions nécessaires lorsqu'une comparaison entre l'ensemble des deux processus est effectuée (Goldman 2004, p. 218 pour les flagellations des initiés cubeo ; Hugh-Jones 1979, pp. 64-65 pour les Barasana ; Hill 2004, p. 33 pour une interprétation de la même action chez les Baniwa, comme une identification du corps des initiés au découpage du palmier issu du corps du héros mythique). C'est en tout cas dans ce sens qu'il faut comprendre les affirmations suivantes :

"In doing this, that which I mentioned [ poreró] came into existence, like the cockroach, the miuu tree, and the botfly ". Kahsië: cockroach, this word is related to the term kahséro/shell, peel, bark. It is said of cockroaches that they " change their skin " much like snakes or certain river crabs, in which is seen symbolism of renewal and transformation. [...] miuu; this is the name of a tall forest tree, [...]. [...] the true symbolic importance of this tree lies in the fact that it occasionally sheds large peaces of bark, revealing underneath a smooth, new surface of a yellowish colour. The tree, then, is another symbol of renewal. [...] Behkóë is the name of the common horsefly (Dermatobia hominis). This stinging insect symbolizes impregnation (see Reichel-Dolmatoff 1971, pp. 58-59 passim). [... I In sum, the three agents invoked in the spell represent the power of periodic renewal and of impregnation. (Reichel-Dolmatoff 1996, p. 85, I, §155)

Il convient de revenir brièvement sur l'identification proposée par ReichelDolmatoff de l'insecte, désigné par le terme behkóë, pour montrer que c'est bien la guêpe parasite et son mode de reproduction qui sont le modèle de base de 
l'ensemble du complexe rituel de Yurupari. Reichel-Dolmatoff' (ibid., p. 86) identifie l'insecte comme étant Dermatobia hominis, c'est-à-dire un insecte diptère qui, sous sa forme larvaire, est un parasite de l'homme (mais aussi des autres mammifères, aujourd'hui principalement du bétail). Dans ses commentaires, cet auteur le désigne comme le taon commun ( "common horsefly »), ce que Dermatobia hominis n'est pas puisqu'il appartient à la famille des cuterebridae (Hogue 1993, p. 401), alors que le taon appartient à une autre famille (celle des tabanidae, Hogue ibid., p. 383). Notons que, parasite sous sa forme larvaire, cette espèce ne pique pas l'hôte qu'elle va habiter, mais se sert de n'importe quel autre insecte " piqueur » comme vecteur de ses œufs qu’elle aura préalablement posés sur eux (Hogue ibid., p. 401). La larve, déjà largement développée dans la capsule de l'œuf, profite de la petite plaie occasionnée par l'insecte vecteur pour pénétrer la peau de l'hôte et s'y maintenir en le parasitant. Le ver ainsi généré est communément désigné par muche ou munche en espagnol local. Il est toutefois largement distingué du taon par les populations indigènes de la région, puisqu'il se retrouve comme parasite des chiens, du bétail et, pour cette espèce particulière (Dermatobia hominis), des hommes, alors que le taon n'est pas parasite (en tous cas les populations indigènes de la région font cette distinction). De plus, le taon pique grâce à ses appendices buccaux, ce qui n'est pas ignoré par les Indiens, puisque l'action est décrite comme une ponction, et non grâce à un dard dont il est dépourvu. Dans la métaphore « inséminatrice »évoquée par Reichel-Dolmatoff, c'est bien l'abdomen et le dard qui, dans une compréhension anthropomorphique, sont interprétés comme l'équivalent d'un phallus - l'œuf et la larve étant interprétés comme le rejeton de ce "mâle », bien qu'étant issus d'un individu femelle. Cette compréhension anthropomorphique, spécifique aux groupes de cette région, annonce d'ailleurs l'idéologie largement patrilinéaire : l'enfant " provient » du père (Karadimas 2003; Jara 1996). Pour rester dans le registre entomologique, il existe dans ce groupe des diptères (mouches, taons...) un représentant de la famille des mydidae (Mydas rubidapex, Hogue 1993, p. 385) qui imite un autre insecte, en l'occurrence une très grande guêpe solitaire Pepsis heros (un pompilidae) qui possède le comportement parasitö̈de, le dard et la qualité " brûlante " provoquée par sa piqûre. Pour les Miraña, cette guêpe est la personnification du héros culturel miraña Souffleur-de-Sarbacane; elle fait une piqûre affreusement douloureuse, qui donne véritablement une impression de brûlure intense.

\section{YuRuPari ET Kuwal, des guêpes ParastToÏDES}

C'est vers ce dernier insecte qu'il faut rapprocher l'identification dans la mesure où le quatrième texte du recueil Yurupari de Reichel-Dolmatoff fait explicitement référence à une guêpe solitaire, à une guêpe parasitoïde. Toutefois, 
d'après Reichel-Dolmatoff, celle-ci est appelée au secours de la narration pour son caractère brillant :

"They burned that komé mahsá bëgë with that fire; they say [that] they destroyed the komé mahsá bëgë ». komé mahsá bëgë, lit. " Metallic people old time creature », a name given to a large black and yellow wasp which is characterized by a bluish " metallic » color : [...] A pertinent comment was : "That wasp is always looking for fresh meat to lick; it is always after woman 》. It is said : "They destroyed that poreró ". This wasp can thus be equated with the mantis (bari sero) and the cricket ( poreró). (ReichelDolmatofi 1996, p. 243, IV, §1)

Ici, le mythe commence par la destruction de cette guêpe solitaire, d'aspect métallique et brillant ${ }^{14}$. Or je viens de faire remarquer que cette guêpe était justement un modèle inséminateur particulier : elle pond ses œufs sur ses victimes - d'autres insectes paralysés - qui se font dévorer ou sucer par les larves lors de leur éclosion. Comme nous venons de le voir dans la citation, les insectes chassés et paralysés sont perçus comme les femmes de cette guêpe ou, dans le cas des Miraña, comme des ennemis vaincus (Karadimas 2003). Chaque descendance implique une nouvelle victime, c'est-à-dire une « mère » par enfant. Or c'est ce modèle de sexualité qui est ici remis en question puisque, avec l'avènement du modèle «chrysalide », il y a renouvellement de la peau qui entoure l'enfant (placenta et membrane amniotique) pour chaque nouvelle naissance en lieu et place d'une nouvelle «mère ». La limitation de ce modèle est qu'il sera intrautérin pour que l'enfant soit nourri par un tube - le cordon ombilical reliant l'enfant au placenta -, comme dans le modèle de la chrysalide où le pédoncule sert d'élément suspenseur et est figuré, sur la trompe de Yurupari, par l'embout.

Rappelons que, chez les Miraña, les guêpes parasitö̈des sont un modèle pour évoquer la sarbacane. De plus, trompe et sarbacane sont, chez les Miraña, des modèles complémentaires : l'un devant contenir l'autre comme la chrysalide contient le papillon, ou l'exuvie contient la guêpe (chez les Baniwa, des fléchettesesprits sont projetées par l'embout des flûtes, laissant entrevoir l'association entre flûtes et Kuwai qui est une guêpe, voir infra, Wright 1993, p. 11). Il semble que, dans le quatrième texte du recueil de Reichel-Dolmatoff, la guêpe occupe la même place que Tonnerre dans le premier, ainsi que la "chose brillante » des deux autres textes (le quartz et l'élément brillant placé dans une cavité d'arbre). De plus, l'aspect brillant/métallique de cette guêpe recouvre une des phases de la métamorphose chez les chrysalides des papillons (c'est d'ailleurs l'étymologie du terme : en grec, chrysalide signifie « la chose dorée »).

Cette guêpe parasitoïde est également présente dans la tradition des Cubeo, autre groupe de la région qui, bien que de langue arawak, est à rattacher à l'ensemble culturel du Nord-Ouest amazonien dominé par les groupes de langue tukano. Dans un des récits faisant référence à la création des trompes de Yurupari, le personnage Lune (Komí), après avoir dévoré trois des enfants de son frère 
Aínyehinkü qui lui ont désobéi alors qu'il leur enseignait « toutes les choses du monde ", s'enfuit dans le ciel parce que son frère cherchait à le tuer. Le plus jeune des enfants de Aínyehinkü veut savoir ce à quoi ses frères n'ont pu accéder par désobéissance :

He thought that he might get Komi to come down again. But Komi was now afraid. Ainyehinkü therefore sent a wasp [uchíkü] to the sky to fetch him. Uchíkü brought Komí an offering of tree larvae [ari ava] and was pleased. Having eaten it, he looked down at the earth with satisfaction and saw they were blowing büküpwanwa [trompes de yurupari] to bring him down. (Goldman 2004, p. 203)

Dans un texte précédent, c'est déjà un type similaire de guêpe qui apparaissait afin d'envoyer une pièce de gibier en offrande (Goldman ibid., p. 199). Cet épisode existe également chez les Baniwa/Wakuenai avec le personnage de Kalimátu, une guêpe qui apporte une larve de Rhynchophorus à Kuwai (Hill 1993, p. 65 , 2004, p. 29 ; Wright 1993, p. 10). Dans la mythologie cubeo, Komí descendra en faisant sonner son corps comme le ferait une trompe et sera ensuite brûlé non sans avoir enseigné comment guérir les maladies : en mémoire de son sacrifice naîtront les palmiers paxiuba (Iriartea deltoidea et surtout Socratea exorrhiza) qui seront là pour rappeler son image et le son qu'il produisait. Remarquons ici que les guêpes pompiles, les scolies, mais aussi d'autres comme les guêpes maçonnes (Trypoxylon spp.), produisent avec leurs ailes un vrombissement caractéristique, lequel, particulièrement bruyant et notable, est à mettre en parallèle, me semble$\mathrm{t}$-il, avec le son produit par les trompes ${ }^{15}$.

Ce rapprochement est fait par les Indiens eux-mêmes : dans un mythe baniwa sur l'origine des flûtes sacrées, Kuwai surprend quatre enfants en train de jouer avec des bourdons. Ils enferment les hyménoptères dans une poterie et dansent au son produit, prétendant entendre ainsi la musique des instruments "sacrés ». Kuwai se moque d'eux en leur faisant comprendre que lui seul incarne et produit le véritable son de ces instruments (Wright 1993, p. 10). Un son que l'on peut donc légitimement associer à un bourdonnement d'hyménoptère, ainsi que cela est dit dans un autre mythe de la même population, recueilli par Jonathan Hill (1993, p. 65) : "Then Iñápirrikuli taught Hérri how to create Kuwai's song by putting a bee inside a basket. The buzzing sound was exactly like Kuwai's singing 》.

Rappelons que les seules guêpes qui transportent des larves d'autres insectes comme proies sont des guêpes parasitoïdes. Les insectes transportés sont des larves de coléoptères qui se trouvent dans des troncs ou dans le sol. Le point essentiel à retenir est que ces larves servent de nourriture à la descendance de ces guêpes. Ces dernières confectionnent des abris dans le sol ou dans les anfractuosités ou encore, tels des potiers, dans des cellules de boue dans lesquelles elles transportent les larves paralysées (d'où leur nom anglais de potter wasp). Elles pondent ensuite un œuf qui, transformé en larve carnassière, se nourrira de l'insecte en le suçant, lequel insecte sera interprété par certaines populations 
amérindiennes comme jouant le rôle de «mère ». En d'autres termes, celui qui, dans l'environnement, se nourrit des larves transportées par une guêpe n'est autre qu'une larve de guêpe, c'est-à-dire sa descendance. Le personnage du mythe, Kuwai (ou Komí, Lune ${ }^{16}$ ), peut être identifié à une larve qui va subir une métamorphose. C'est cette larve, entrant dans le processus de métamorphose, qui devient le modèle des trompes ${ }^{17}$. Si elle arrive en vrombissant de tout son corps, c'est que la métamorphose a été accomplie ${ }^{18}$. Ce « bourdonnement », attribué en tant que propriété du corps de Kuwai - personnage équivalent à Yurupari dans la mythologie baniwa/wakuenai -, doit être mis en parallèle avec celui réalisé par les flûtes lors des rituels.

Dans son étude de 1905 publiée en 1909-1910, Koch-Grünberg reproduit un dessin exécuté par un Cubeo dans lequel différents héros culturels sont représentés. Parmi ceux-ci apparaît Hömänhîkö (probablement le même qui, chez Goldman, est désigné sous le nom Aínyehinkü, voir supra), accompagné de Kúai ${ }^{19}$ : ce dernier, selon l'auteur, est doté d'un phallus en érection qui le caractérise "lui comme son frère, en tant que héros de la fertilité » (Koch-Grünberg 1995, vol. II, p. 158 ; traduction de l'auteur D. K. ; Figure 11). Sur le dessin, il est dépeint comme portant un masque à bout de bras puisqu'il est associé aux rituels masqués (Figure 11d). Dans un article récent (Karadimas 2007), j’ai montré comment, chez les Miraña, le Maître des animaux, personnifié par une guêpe parasitoïde, et son costume-masque, doté d'un phallus, pouvaient être associés, par des attributs similaires à ceux de Kúai/Yurupari, à ce même ensemble significatif et à son caractère fortement sexualisé.

Remarquons que, chez les Cubeo et les Baniwa, ce personnage sera brûlé : de ses cendres vont naître des palmiers avec lesquels seront confectionnés les flûtes et les embouts des trompes. C'est également le cas chez les Barasana (Hugh-Jones 2001, p. 251) et dans tous les groupes tukano. Or, dans le quatrième texte desana présenté par Reichel-Dolmatoff, c'est précisément par la crémation de la guêpe parasitoïde que débute la narration. Il faut donc en déduire qu'en tant que modèle général la guêpe parasitoïde cumule les caractéristiques des trompes de Yurupari : larve/cocon, métamorphose et vrombissement (Figure 12).

Enfin, un dernier moyen de lever l'ambiguïté est de se référer à l'ethnographie makuna publiée récemment (Åhrem et al. 2004) dans laquelle on trouve un important recueil de mythes, de traditions et de narrations makuna. Dans le mythe de la Création, la figure du Lombric occupe, selon les dires même des Makuna, une place prépondérante au côté de la Femme-Chamane-créatrice-dumonde (Rõmikimu) : « [...] les lombrics sont très importants dans la création du monde puisqu'ils sont un des composants les plus sacrés qu'il y ait dans l'histoire " (ibid., p. 443 ; traduction de l'auteur D. K.). Ce personnage féminin, présent aussi chez les Barasana, crée Waiyaberoa « Bourdon-du-Pirá »-en fait Yurupari -, " [...] qui, en même temps, était Gütãrotoro "celui qui fait les pierres" » (ibid.). En tukano toujours, beroa - ou berua en barasana (Hugh-Jones 


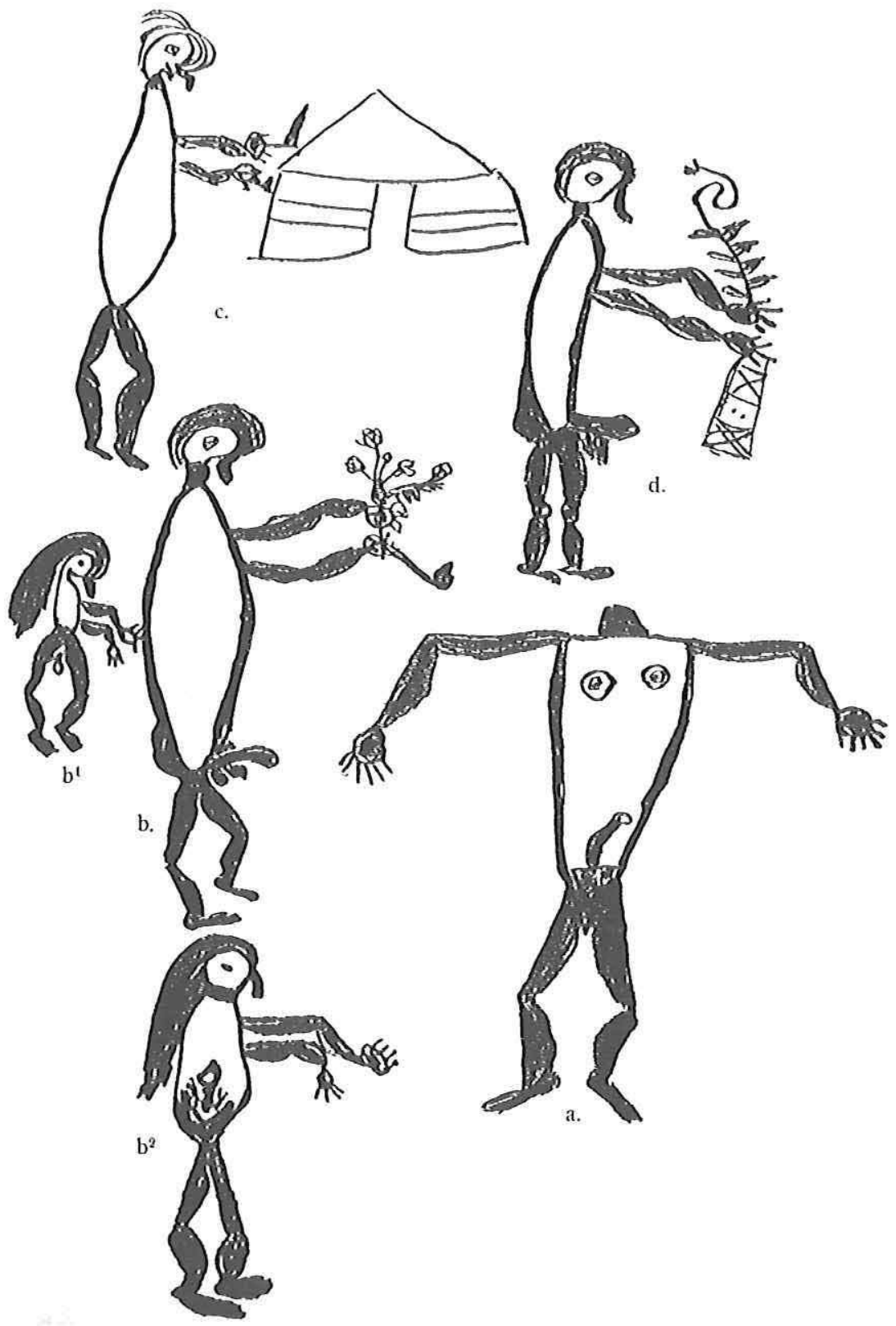

FIG. 11 - Dessin réalisé par un Cubeo figurant différents héros culturels, dont Kúai (d) qui, ici, tient un masque à bout de bras (d'après Koch-Grünberg 1909-1910, figure 93). 


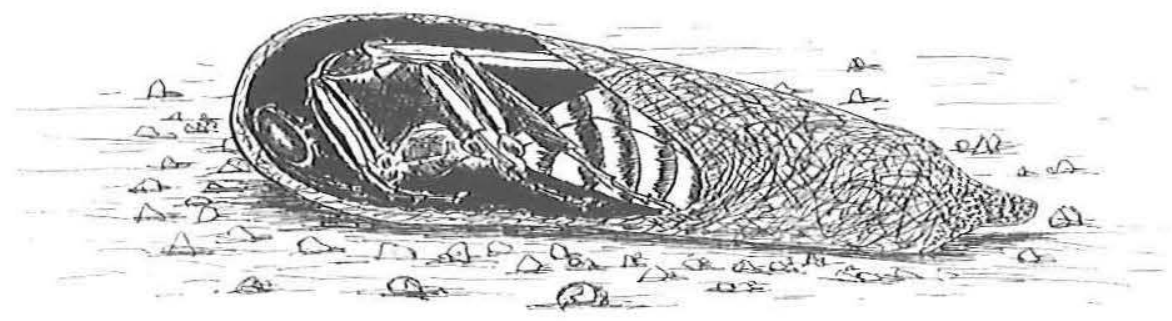

FIG. 12 - Guêpe parasite au moment de sa nymphose, après que la larve ait tissé un cocon : l'ensemble serait figuré par le rituel de Yurupari et les trompes (dessin de l'auteur, D. K.).

1979, p. 164) - est un des trois termes pour désigner les abeilles, que Hugh-Jones distingue des guêpes (utia). Mais le terme abejón désigne le bourdon en espagnol, c'est-à-dire que nous avons plus probablement à faire à un hyménoptère solitaire qu'à un insecte vivant en société. Chez les Miraña, lorsque l'espagnol est utilisé, le terme abejón sert surtout à désigner les espèces solitaires qui produisent un fort bourdonnement lors de leur déplacement ; soit un bourdon, soit, comme dans le texte IV proposé par Reichel-Dolmatoff, une guêpe solitaire.

Les Makuna précisent que "Waiyaberoa est le sauvage, itinérant et vagabond, qui ne vit pas sur un seul lieu : c'est pour cela qu'il lui arrive toujours quelque chose» (Åhrem et al. 2004, p. 90 ; traduction de l'auteur D. K.) ; un personnage placé plutôt du côté de l'agressivité et du caractère solitaire, donc. Chez les Makuna aussi, Yurupari serait un hyménoptère et, fort probablement, une guêpe parasite, mais on ne peut encore l'affirmer avec certitude. Si l'on rajoute à cela qu'il était également " celui qui faisait les pierres » (voir supra ; ce personnage mythique a, en plus, la propriété de les « cimenter », ibid., p. 97), il pourrait alors s'agir d'une espèce d'hyménoptère qui façonne des pierres ou qui " maçonne ", comme une guêpe parasite ${ }^{20}$.

Avant que ce personnage ne fût créé par la Femme-Chamane, celle-ci, alors que rien n'existait encore, fit apparaître Kũrükuiĩno « Anaconda-de-la-bouturede-manioc » (personnage identique à Manioc-stick-Anaconda chez les Barasana ${ }^{21}$ ) qui n'est pas son fils. Il apparaît que les deux personnages, Bourdon-duPirá et Anaconda-de-la-bouture-de-manioc, sont les flûtes de Yurupari et ne forment finalement qu'un seul et même personnage : « Waiyaberoa et Kũrüküĩno sont un seul, et sont le même corps, mais la transformation eut lieu lorsque ses apprentis le brûlèrent considérant qu'il était mauvais. Il cessa d'être une personne pour être les grandes palmes que les dieux ont coupées en morceaux pour donner 
la forme actuelle aux instruments: toutefois, le Yurupari continue d'être Kũrüküĩno » (Åhrem et al. 2004, p. 91 ; traduction de l'auteur D. K.). Il s'agit donc du même personnage : une guêpe solitaire aux reflets bleus métalliques qui, dans le texte IV (desana) proposé par Reichel-Dolmatoff, sera brûlée et dont les cendres donneront naissance au palmier paxiuba.

Il est donc possible d'affirmer que le complexe de Yurupari est une mise en scène rituelle et une application socio-sexuelle rendant accessible, à travers la mythologie, la compréhension de l'ensemble du processus de reproduction d'une guêpe parasitoïde et ce non seulement pour les Desana et les Makuna mais également pour les autres groupes tukano comme les Barasana, les Cubeo et les Baniwa/Wakuénai de langue Arawak. Dans une certaine mesure, ce modèle dégagé pour les groupes du Vaupès lato sensu est également valide pour les groupes du Caquetá/Putumayo, c'est-à-dire les Miraña et Uitoto.

Il resterait, avant de conclure, à comprendre en quoi le palmier paxiuba doit son origine à cette guêpe. Plus spécifiquement, il faut s'interroger sur les raisons qui font que ce palmier naît des cendres de la guêpe.

En examinent attentivement les propriétés de ce palmier, il en est une qui semble avoir échappé à Reichel-Dolmatoff. Le paxiuba se multiplie par sexuation (donc par fructification). Toutefois, dans le cas où un arbre viendrait, en tombant, à se coucher contre terre, son " tronc » (le stipe), suffisamment souple, se pliera mais restera ancré grâce à ses racines échasses, là où d'autres palmiers seraient simplement déracinés et condamnés. À partir de l'endroit du stipe le plus proche du sol, le paxiuba va projeter de nouvelles racines échasses et redresser sa frondaison pour poursuivre sa croissance. Il procède ainsi à une sorte « d'autobouturage ». Mais il y a plus.

Chez les Barasana, l'ensemble des flûtes et des trompes du rituel forme le corps de Yurupari. Les flûtes sont ses « jambes » et ses «bras », alors que les trompes forment son « corps ». Or ce corps est celui de la guêpe parasite, soit sous forme larvaire, soit sous forme adulte. $\mathrm{Si}$ je reprends une théorie plus générale de la génération des savoirs à partir des images mentales générées par la perception sur laquelle je travaille depuis plusieurs années, il apparaît que le palmier plié et qui a jeté de nouvelles racines échasses possède, en association avec sa frondaison de palmes et le racème de fruits, le même schéma d'image que la guêpe parasite (l'un et l'autre sont appréhendés de façon similaire par le cerveau humain). Les racines échasses, hérissées de petits piquants, correspondent à l'image des longues pattes de la guêpe, le stipe recourbé à l'abdomen, la frondaison de palmes aux ailes bruissantes, le racème, avec ses bractées bipennées, à la tête et, enfin, une des racines sortantes du premier ensemble de racines-échasses correspondrait au dard-pénis de l'hyménoptère : il s'agit en fait d'une correspondance par l'image (Figure 13).

Si j'associe maintenant ces propriétés « d'auto-bouturage », dégagées plus haut, au fait que la guêpe est également, avant sa nymphose, une larve et que c'est 

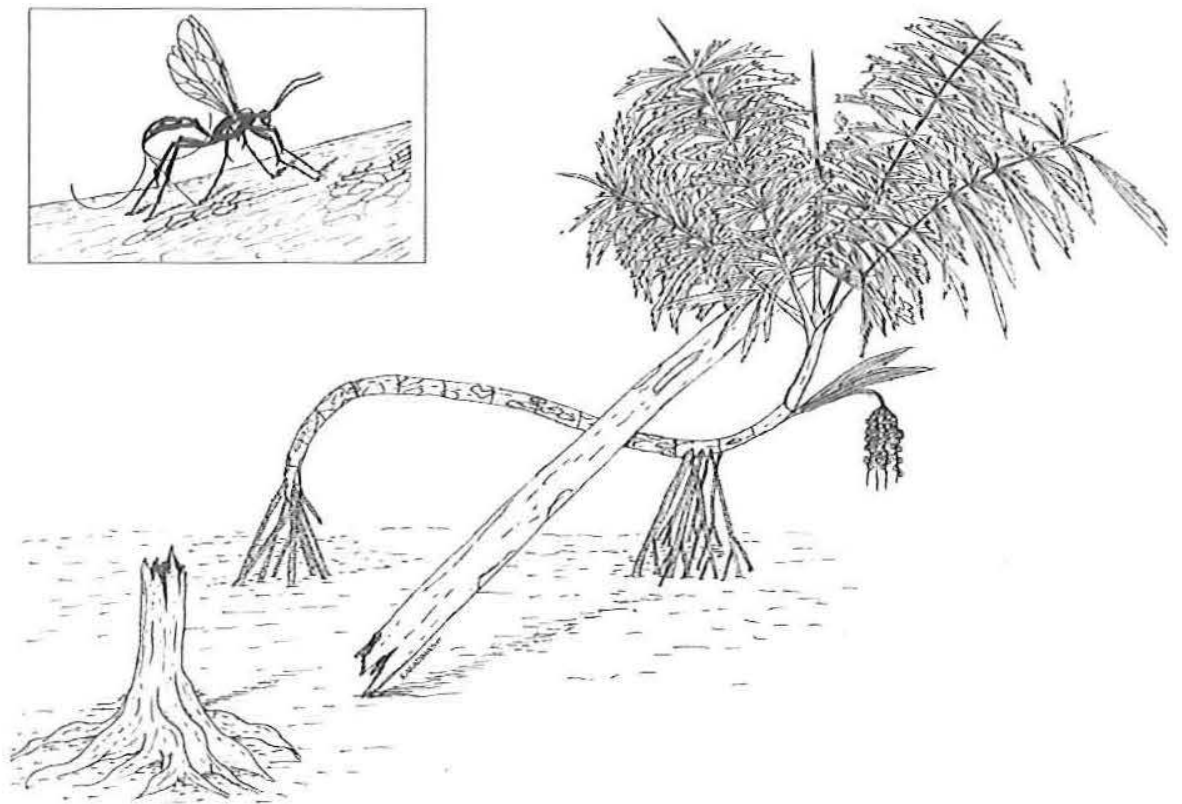

FIG. 13 - Le palmier Socratea exorrhiza comme image de la guêpe parasite (dessin de l'auteur, D. K.).

bien ce personnage qui est décrit dans le mythe de Yurupari, il faut revenir sur son nom «Anaconda-de-la-bouture-de-manioc » ou " Manioc-stick-Anaconda » chez les Barasana décrits par Hugh-Jones. S'il s'agit du même personnage que la guêpe parasite chez les Desana ou le "Bourdon-du-Pirá » des Makuna, il faut reconnaitre que ce sont là deux désignations qui se réfèrent au même être, avant et après sa métamorphose. En association avec la qualité de « bouturage » dégagée pour le palmier, remarquons que la description convient pour le qualifier "d'Anaconda-de-la-bouture-de-manioc ». En d'autres termes, il est "fiché », planté, comme on bouture le manioc. Il renaît de lui-même en étant planté. Cette description nous fait revenir au point de départ de cet article, avec la création du monde chez les Miraña, chez lesquels le monde apparaît comme "fiché ", planté ${ }^{22}$.

Une solution distincte de cette identification est de reconnaître que la désignation "Anaconda-de-la-bouture-de-manioc » se réfèrerait à un prédateur " ophidiomorphe » du manioc, plus exactement de ses feuilles et/ou branches (i.e. " bouture de manioc ", puisque le bouturage se réalise par sectionnement de la tige d'une plante adulte qui est replantée). D'aspect ophidien, le prédateur du manioc devrait également être associé à des guêpes parasites. Le destructeur principal de Manihot esculenta est une grande chenille d'un sphingidé, Erinnyis ello, qui attaque la plante et fait subir, lorsqu'elle pullule, des dégâts considérables 


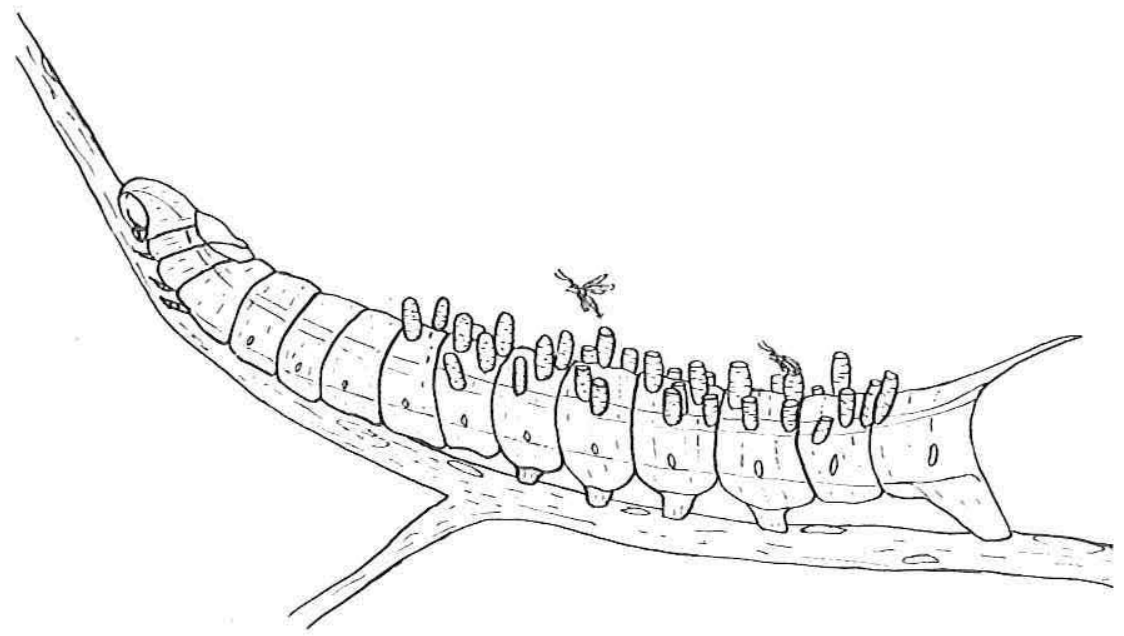

(a)

(b)

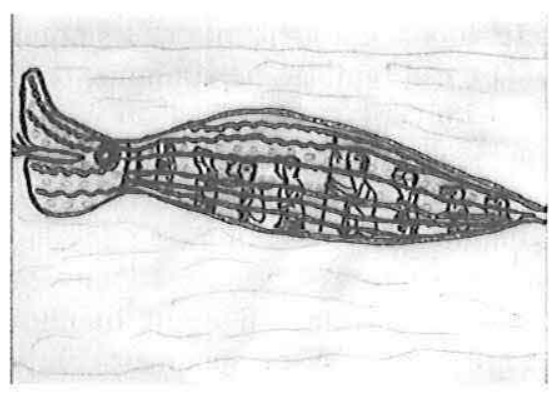

FIG. 14 - Mise en parallèle de représentations de l'« anaconda-de-transformation » ou " anacondacanoë » réalisées par les Indiens Desana (dessins de l'auteur, D. K. ; d'après Pàrõkumu et Kèhíri 1995, figures 8 et 9) et d'une chenille parasitée par une guêpe dont les larves, après avoir incubé dans le corps de la chenille, émergent de celui-ci et forment chacune un cocon. 
aux plantations. Plusieurs espèces de guêpes parasitent la chenille en implantant des larves dans son corps. Ces larves y incubent et en émergent sous la forme d'une multitude de cocons attachés à la chenille dans lesquels elles se métamorphosent (Figure 14a). Chacun de ceux-ci donnera naissance à une guêpe adulte ; la chenille, elle, périra, non sans avoir donné l'impression de les transporter dans son corps, puis de les en avoir fait émerger et naître. Ce dernier point nécessiterait de plus amples informations ethnographiques car il a des implications dans la mythologie vaupèsienne et, plus particulièrement, en ce qui concerne la figure de " l'anaconda-canoë » (Figure 14b) ou " anaconda-de-transformation » qui a transporté dans son corps les premiers ancêtres claniques (Figure 14c) et les a fait émerger dans leur " maison de naissance » en suivant un ordre qui respecte la segmentation de son propre corps (C. Hugh-Jones 1979, p. 261 et passim).

\section{CONCLUSION}

Les deux modèles exposés concernant l'origine des flûtes chez les groupes du Vaupès et chez les Miraña renvoient chacun à l'origine de la sexualité sous le même aspect. Dans les deux cas, il existe, à l'origine, une indistinction sexuelle ou, plus spécifiquement, un item qui les subsume, soit de façon concomitante dans le cas des inflorescences du palmier pour la mythologie tukano, soit de façon successive comme dans le cas de la métamorphose d'une chenille/larve en imago pour la mythologie miraña. La différenciation sexuelle est construite à partir d'une partition du « bois » qui fait le clitoris et le pénis. Les parties disjointes se retrouvent de nouveau conjointes dans le rituel de Yurupari, mais manipulées cette fois-ci uniquement par les hommes.

Ce qui semble être souligné, dans un premier temps, dans la représentation que donnent les groupes du Vaupès des éléments du récit de Yurupari est l'association entre les flûtes et les fonctions procréatives, rendues possible grâce à une complémentarité sexuelle. En revanche, chez les Miraña, il s'agit d'une complémentarité contenue dans la continuité d'un même être qui subit des métamorphoses successives. Dans chacune d'elles, l'état des différentes phases est comparé à un état, un organe ou une composante de la reproduction humaine (la chenille qui tisse son cocon est comparée à un pénis et à un flot de sperme qui se solidifie ; ce cocon est une image de la matrice et la pupe qu'il contient est un équivalent du foetus enveloppé dans la membrane amniotique et le placenta). Cette image de la génération extra-utérine est fournie par la pupe dans son cocon. L'enfant brillant qui en émerge ou l'initié est à comparer à l'imago d'un insecte ou, plus précisément, d'une guêpe devenue adulte. Ce que les femmes ont perdu est cette faculté d'engendrer en dehors de leur corps - et le rite de passage de Yurupari permet aux jeunes adultes mâles de l'acquérir. Ce que les hommes ont gagné est cette même faculté, mais il faut désormais engendrer matériellement les enfants des deux sexes à travers le ventre des femmes. 
Cette succession transformationnelle d'un être, même si elle emprunte le langage de la sexualité dans les images qu'elle génère, n'implique plus la complémentarité réelle des sexes pour la création rituelle d'un homme: il n'a pas à repasser dans une matrice, si ce n'est celle, artificielle, que lui tendent les autres hommes. *

* Manuscrit reçu en juillet 2007 et accepté pour publication en mars 2008.

\section{NOTES}

Je tiens à exprimer ma grattitude à Philippe Erikson et Jean-Pierre Goulard pour leur lecture. Je souhaiterais souligner l'importance des commentaires de Stephen Hugh-Jones lors de l'élaboration de ce texte. Il me tient à ceur de remercier chaleureusement Claude Lévi-Strauss pour sa lecture et sa note d'encouragement à poursuivre cette étude au-delà de l'aire culturelle du Nord-amazonien. Il me faut signaler les nombreuses discussions avec Elizabeth Reichel ainsi qu'avec les participants du séminaire « Corps et affects » dirigé par Françoise Héritier qui m’ont permis de compléter certaines de mes approches.

1. Pour une approche comparée des systèmes de connaissance liés à chacun des sexes dans le Nord-Ouest amazonien, voir Reichel 1999.

2. Je présente ici du matériel ethnographique miraña issu de mes premiers terrains (1992-1993) ainsi que d'un terrain complémentaire en 2001 à Léticia, Colombie. Ce dernier séjour a pu être financé grâce à une aide de la fondation Fyssen.

3. Mais aussi correspondre avec les données présentées et publiées par les Indiens eux-mêmes comme par exemple Diakuru et Kisibi 2006, ou Pãrōkumu et Kèhíri 1995, deux importants recueils de mythologies desana.

4. Certaines données présentées ici ont déjà été publiées dans mon ouvrage de 2005.

5. On pourrait comparer cet aspect à ce qui circulerait à l'intérieur d'une bouteille de Klein, passant alternativement de l'intérieur à l'extérieur sans jamais être véritablement contenu.

6. En effet, langage imagé, la narration mythique n'est nullement obligée de réintroduire la comparaison ; elle ne fait que réaflirmer l'analogie par l'identité.

7. Il faut ici remarquer que cette association entre insufflateur de tabac en poudre et phallus est attestée chez les Desana pour lesquels, d'après Reichel-Dolmatoff (1996, p. 39) : " To this we must add that ehéri-goá means "marrow bone", one of the many' designations of the phallus and also the name of the tubular smuffing apparatus, or bird bone, through which Desana shamans absorb a hallucinogenic powder ». En revanche, cet auteur ne fait aucun cas du contenant en coquille d'escargot qui l'accompagne. Dans un de leurs mythes, les Barasana soulignent en parlant des gens $\mathrm{He}$, c'est-à-dire des trompes, que : "The snail-shell snuff holder was their shamanism » (Hugh-Jones 1979, p. 265), c'est-àdire que ces coquilles de gastéropodes sont à l'image des trompes. Christine Hugh-Jones ne propose pas d'analyse du contenant à tabac en poudre fait dans une coquille d'escargot.

8. Bien que j'utilise cet ensemble de textes comme point central de mon étude, je ne concorde pas entièrement avec les analyses qu'en propose l'auteur (voir infra).

9. Ainsi l'image de la presse à manioc comme un outil de transformation et d'engendrement extra-utérin est également présente dans d'autres mythologies amazoniennes. Dans un mythe ayant trait, selon Carvalho $(1979$, p. 90$)$, à un cycle de transformation comparable à celui de Yurupari (légende de « Pahy-Tuna » (?) en provenance du Rio Branco), la presse à manioc sert d'instrument pour transformer un enfant couvert de pustules; la mère " prit un tipity, y mit l'enfant, le suspendit, [passa une perche pour faire levier et] s'assit à l'autre bout, serrant ainsi son propre enfant dans le tube. Les humeurs qui en sortirent furent telles que, quand elle retira l'enfant, celui-ci avait subi une métamorphose complète. C'était l'enfant le plus joli qu'on pût voir... " (Sant'Anna Néry 1889, p. 154, in 
Carvalho 1979, p. 90). Dans le même ouvrage, la variante combinatoire que propose Carvalho de l'enfant ainsi « exprimé » est justement un cutia (un acouchi) en tant que Maître de l'arbre à manioc, variante qu'elle emprunte à l'étude des Cubeo réalisée par Goldman (1963). Ici encore, c'est à l'image de la chrysalide suspendue que fait penser l'action de placer un enfant dans la presse à manioc afin de lui faire subir une métamorphose.

10. Pour Stephen Hugh-Jones (communication personnelle), seule l'identification à une grosse sauterelle amazonienne correspond à bari-sero. Selon lui, il n'existe pas, en dehors des informations fournies par Reichel-Dolmatoff, d'indications qui permettent d'identifier bari sero à une mante religieuse.

11. Pour une discussion complète sur les enfants dits " nés-coiffés », voir Belmont 1971.

12. J'ai présenté récemment une construction similaire pour le conte du Petit Chaperon rouge dans lequel la pièce de vêtement, le chaperon, est en fait une évocation du capuchon clitoridien (Karadimas 2004).

13. Il convient de faire remarquer que Georges Devereux, dans son ouvrage La vulve mythique (1983), revient sur le cas d'une femme chamane lesbienne chez les Mohave qui, suite à plusieurs tentatives de séduction auprès de la femme d'un homme, se fait violer par ce dernier. Ici, le viol en acte, et non seulement en menace, se trouve placé comme réponse masculine à l'homosexualité féminine.

14. La description de Reichel-Dolmatoff pourrait laisser penser qu'il s'agit d'une variété de Scolia, grande guêpe parasitoïde (mesurant près de $5 \mathrm{~cm}$ ), noire et poilue, avec des marques jaunes sur la face postérieure de son abdomen, qui possède des ailes à reflets métalliques bleutés (peut-être de la famille des Campsomeris rencontrés en Amérique du Sud) et qui parasite les larves de coléoptères (scarabée). Cette grande guêpe est associée à l'une des formes prises par le Maître des animaux, poilu chez les Miraña. Ce dernier s'attaque aux humains, comme la guêpe le fait pour les larves de coléoptères qui représentent, pour elles, ce que les larves palmistes sont pour les humains (Karadimas 2005, p. 342). Chez les Wakuénai du Venezuela, Kuwai - à identifier comme un hyménoptère, voir infra -, est un être également poilu (Hill 1993, p. 63).

15. Par exemple la description fournie par Henry Walter Bates The Naturalist on the River Amazon (1975, chap. VIII, part. II) est tout à fait symptomatique : "The species of Trypoxylon, however, are all building wasps; wo of them which I observed (T. albitarse and an undescribed species) provision their nests with spiders, a third (T. aurifrons) with small caterpillars. Their habits are similar to those of the Pelopaeus - namely, they carry off the clay in their mandibles, and have a different song when they hasten away with the burden to that which they sing whilst at work. Trypoxylon albitarse, which is a large black kind, three-quarters of an inch in length, makes a tremendous fuss while building its cell. It often chooses the walls or doors of chambers for this purpose, and when two or three are at work in the same place, their loud humming keeps the house in an uproar. The cell is a tubular structure about three inches in length ".

16. Il faut, par ailleurs, faire un parallèle entre cette présence de Lune dans ce cas-ci, traité en renaissance par l'action des trompes et Lune dans la mythologie jivaro présentée plus haut. Dans le mythe jivaro, Lune, enterrée, réussit à renaître suite à l'action d'une trompe faite à partir d'une conque.

17. Dans un article intitulé fort judicieusement « Metamorphosis: mythic and musical modes of ceremonial exchange among the Wakuénai of Venezuela» (2004) qui traite de ce même ensemble mythologique lié au personnage de Kuwai, Hill accorde la même importance que celle présentée dans cet article au processus de métamorphose des insectes comme métaphore générale de ce processus rituel. Pour lui et pour les Wakuénai, ce sont plus les larves palmistes du type Rhynchophorus et leur métamorphose qui sont à la base de cette comparaison que leurs utilisations comme proies/femmes par leurs prédateurs naturels, les guêpes parasitoïdes. Il s'en suit, me semble-t-il, une altération de l'identification " prédatrice » qui serait donnée à la figure de la guêpe et, partant, une forte désexualisation de l'acte qui mène justement Hill (ibid., p. 41) à le considérer pour les Wakuénai comme « [...] a powerfull expression of the idea of a non-sexual form of reproduction ". Cette sexualisation de l'acte d'insémination/prédation revient dès lors que la figure de la guêpe et son caractère belliqueux sont pris en compte. Chez les Barasana en tout cas, "the two men who enter the house at the climax of the $\mathrm{He}$ 
House (yurupari) are fierce spirits [...] who come to teach the initiates to be brave and to kill " (Stephen Hugh-Jones 1979, p. 205 ; souligné par l'auteur, D. K.).

18. Dans une phase rituelle et dans l'épisode mythique qui l'accompagne, cette guêpe pompile représente, chez les Miraña, Lune et/ou « Soleil noir » qui est le soleil souterrain (Karadimas 2003).

19. Koch-Grünberg (1995, p. 156) indique d'ailleurs que ce héros est d'origine baniwa.

20. Une autre raison, pour Stephen Hugh-Jones (1979, p. 206 ; communication personnelle), de reconnaître comme valide cette association entre guêpe parasite et Yurupari est le fait que les verges utilisées pour fouetter les initiés sont appelées heta waso, c'est-à-dire le nom donné aux dards des fourmis tocandira Paraponera clavata (dont la piqûre est douloureuse).

21. Pour une comparaison entre He-Anaconda (Yurupari-Anaconda) et Manioc-stick-Anaconda chez les Barasana, voir C. Hugh-Jones 1979, p. 184.

22. L'ensemble de la comparaison demanderait cependant un autre travail tant les implications sont importantes pour l'ethnographie de la région : je me cantonne ici à présenter l'idée maîtresse.

\section{RÉFÉRENCES CITÉES}

\section{Åhrem Kay, Luis Cayón, Gladys Angulo et Maximiliano García (éds)}

2004 Etnografia makuna. Tradiciones, relatos y saberes de la Gente de Agua, ICANH-Acta Universitatis Gothoburgensis/ICANH, Göteborg/Bogotá.

\section{BATES Henry Walter}

1975 The Naturalist on the River Amazon, Dover Publications, New York.

\section{BeLmont Nicole}

1971 Les signes de la naissance. Étude des représentations symboliques associées aux naissances singulières, Plon, Paris.

\section{Carvalho Silvia Maria S. de}

1979 Jurupari : estudos de mitologia brasileira, Atica, São Paulo.

\section{Devereux Georges}

1983 Baubo, la vulve mythique, Jean-Cyrille Godefroy, Paris.

Diakuru Américo Castro F. et Durvalino Moura F. Kisibi

2006 Bueri Kâdiri Marîriye. Os ensinamentos que nâo se esquecemi, FOIRNUNIRT, Col. « Narradores Indígenas do Río Negro », 8, Sâo Gabriel da Cachœira.

\section{GoLDMAN Irving}

1963 The Cubeo : Indians of the Northwest Amazon, University of Illinois Press, Urbana.

2004 Cubeo Hehenewa religious thought : metaphysics of a Northwestern Amazonian People, édité par Peter J. Wilson, Columbia University Press, New York.

\section{Goulard Jean-Pierre}

1998 Les genres du corps: conceptions de la personne chez les Ticuma de la Haute Amazonie, thèse de $3^{\mathrm{e}}$ cycle, EHESS, Paris.

\section{HeMming John}

1984 En busca de El Dorado, Ediciones del Serbal, Barcelone. 
HiLl Jonathan D.

1993 Keepers of the sacred chants : the poetics of ritual power in an Amazonian society, University of Arizona Press, Tucson/Londres.

2001 "The variety of fertility cultism in Amazonia : a closer look at gender symbolism in Northwestern Amazonia ", in Thomas A. Gregor et Donald Tuzin (éds), Gender in Amazonia and Melanesia: an exploration of the comparative method, University of California Press, Berkeley/Los Angeles/Londres, pp. 45-68.

2004 "Metamorphosis : mythic and musical modes of exchange in the Amazon rain forests of Venezuela and Colombia ", in Malena Kuss (éd.), Music in Latin America and the Caribbean : an encyclopedic history. Vol. 1 : Performing beliefs. Indigenous cultures of South America, Central America and Mexico, University of Texas Press, Austin, pp. 25-47.

Hogue Charles

1993 Latin American insects and entomology, University of California Press, Berkeley/Los Angeles/Londres.

Hugh-Jones Christine

1979 From the Milk River : spatial and temporal process in Northwest Amazonia, Cambridge University Press, Cambridge.

Hugh-JoNEs Stephen

1979 The Palm and the Pleiades. Initiation and cosmology in Northwest Amazonia, Cambridge University Press, Cambridge.

2001 «The gender of some Amazonian gifts : an experiment with an experiment ", in Thomas A. Gregor et Donald Tuzin (éds), Gender in Amazonia and Melanesia : an exploration of the comparative method, University of California Press, Berkeley/Los Angeles/Londres, pp. 245-278.

JARA Fabiola

1996 «La miel y el aguijón. Taxonomia zoológica y etnobiología como elementos en la definición de las nociones de género entre los andoke (Amazonia colombiana) ", Journal de la Société des Américanistes, 82, pp. 209-258.

KaRAdimas Dimitri

2003 «Dans le corps de mon ennemi. L'hôte parasité chez les insectes comme un modèle de reproduction chez les Miraña d'Amazonie colombienne ", in Elisabeth Motte-Florac et Jacqueline Thomas (éds), Les « insectes 》 dans la tradition orale, Peeters, coll. « Ethnosciences » 407, Paris, pp. 487-506.

2004 "Le Petit Chaperon rouge : comment dire le corps sans le nommer? », in Françoise Héritier et Margarita Xanthakou (éds), Corps et affects, Odile Jacob, Paris, pp. 121-135.

2005 La raison du corps. Idéologie du corps et représentations de l'environnement chez les Miraña d' Amazonie colombienne, Peeters, coll. « Langues et sociétés d'Amérique traditionnelle » 10, Paris/Louvain.

2007 "Yurupari ou les figures du diable: le quiproquo des regards croisés », Gradhiva, 6, nouvelle série, pp. 45-58. 
Koch-GrüNBERG Theodor

1909-1910 Zwei Jahre unter den Indianern: Reisen in Nordwest-Brasilien 1903/1905, 2 vol., E. Wasmuth, Berlin.

1995 Dos años entre los indios. Viajes por el noroeste brasileño 1903-1905, Universidad Nacional de Colombia, Bogotá.

LÉvi-Strauss Claude

1985 La potière jalouse, Plon, Paris.

PÃrõKunu Umusĩ [Firmianto Arantes L.] et Tõrãmu KẽHíri [Luiz GoNes L.]

1995 Antes o mundo não existia : Mitologia dos antigos Desana-Kẽhiripõrã, FOIRN-UNIRT, col. " Narradores Indigenas do Río Negro " 1, Sâo Gabriel da Cachœira.

Petersen de Piñeros Gabriele et Eudocio Becerra (Bigidima) (éds)

1994 Konrad Theodor Preuss : religión y mitología de los uitotos. Recopilación de textos y observaciones efectuadas en una tribu indigena de Colombia, Suramérica, Colcultura/COA/Editorial Universidad Nacional, 2 tomes, Bogotá.

Preuss Konrad Theodor

1921-1923 Religion und Mythologie der Uitoto. Textaufnahmen und Beobachtungen bei einem Indianerstamm in Kolumbien, Südamerika, Vandenhoeck et Ruppecht, Leipzig, J. C. Hinrichs'sche Buchuhandlung, 2 Bände, Göttingen.

RAMIREZ Henri

1997 A fala tukano dos Yépâ-Masa (Tomo I : Gramatica ; Tomo II : Diccionario ; Tomo III : Método de aprendizagem), Inspetoria Salesiana Missionaria da Amazônia/CEDEM, Manaus.

ReICHeL D. Elizabeth

1999 "Cosmology, worldview and gender-based knowledge systems among the Tanimuka and Yukuna, Northwest Amazon ", Worldviews : Environment, Culture and Religion, 3 (3), pp. 213-242.

\section{REICHEL-Dolmatoff Gerardo}

1996 Yurupari : studies of an Amazonian foundation myth, Harvard University Press, Cambridge, Massachusetts.

1997 Chamanes de la selva pluvial. Ensayos sobre los Indios Tukanos del Noroeste Amazónico, Themis Books, London.

\section{STRADELli Ermanno}

1890 "Leggenda dell' Jurupary », Bolletino della Società Geographica Italiana, ser. 3, 3 (27), pp. 659-689, 798-835.

Wright Robin

1993 «Pursuing the spirit. Semantic construction in Hohodene Kalidzamai chants for initiation ", Amerindia, 18, pp. 1-40. 